\title{
An evidence map of digital tools to support social engagement in older adults living with mental illness or those who are at risk for mental health decline
}

\author{
Ellinor Larsson $\mathrm{PhD}^{\mathrm{a}, *}$, Gunilla Fahlström $\mathrm{PhD}^{\mathrm{b}}$, Göran Bertilsson $\mathrm{PhD}^{\mathrm{b}}$, Sofi Fristedt \\ $\mathrm{PhD}^{\mathrm{c}}$ \\ ${ }^{a}$ Department of Health Sciences, Luleå University of Technology, Luleå, Sweden; ${ }^{b}$ Swed- \\ ish Agency for Health Technology Assessment and Assessment of Social Services (SBU), \\ Stockholm, Sweden; 'Jönköping University, Department of Rehabilitation \& ARN-J Aging \\ Research Network, Jönköping, Sweden; *Corresponding author: ellinor.larsson@ltu.se
}

\begin{abstract}
Background: The use of digital technology might contribute to social engagement and mental health in an ageing population.

Objective: To systematically map and describe the scientific knowledge and knowledge gaps on the effects of different types of digital tools for outcomes related to social engagement in older adults at risk for mental health decline.

Methods: Thirteen groups of outcomes (domains) were defined, related to social engagement, by the use of digital tools. Thereafter, a systematic literature search was conducted in April 2017 to identify systematic reviews in line with the objective, an additional search for systematic reviews published since April 2017 was done in September 2019. In addition, reference lists as well as protocols from the Cochrane Collaboration and Campbell were searched. The final sample includes nine systematic reviews, published between 2012 and 2019, with both qualitative and quantitative results.

Results: This evidence map shows that education in and use of digital tools, including computers and the internet, might decrease loneliness. Research is still limited, but the existing research points towards positive effects from the use of digital tools on outcomes related to social engagement, such as loneliness, social isolation, social interaction and/ or social support, participation, depression, physical health/activities, as well as security, self-esteem or empowerment. Scientific knowledge gaps are present for the domains of quality of life, activities in daily living (ADL), cognition, autonomy, and usability.

Conclusion: The evidence map shows inconclusive evidence about the effects on outcomes related to social engagement from the use of digital tools for older adults at risk of mental health decline or mental illness. More research is needed, and future research should include studies with a strong design, larger populations, and various subgroups of older adults in different contexts to further evaluate the effects of the digital tools related to social engagement for older adults at risk of mental health decline or mental illness.
\end{abstract}

Keywords: Information and communication technology, interactive computer games, loneliness

\section{INTRODUCTION}

This study highlights the potential to support older adults' mental health and social engagement through the use of digital technology/tools. Demographically, the number of older adults is rapidly increasing in society; therefore, there is an urgent need to promote maintained physical, but also, mental health as people age. The commonly used definition from 2001 by the World Health Organization (WHO) defines mental health as "a state of well-being in which an individual realizes his or her abilities, can cope with the normal stresses of life, can work productively and fruitfully, and is able to make a contribution to his or her community" (WHO, 2004).
In later life, a decline in mental health, or mental illnesses, as in example depression, can be related to frailty, somatic illnesses, or other life challenging events as part of aging (Vaughan, Corbin, \& Goveas, 2015). In this study, both older persons with medically diagnosed mental illnesses, as well as all older adults at risk of mental health decline (i.e., persons who do not experience mental health or at least experience a decrease in mental health), are included in the population. Interventions are needed to promote mental health in later life, for example supporting participation both in physical and social activities (The Swedish National Institute of Public Health, 2007). 
Table 1. Overview of domains and the included reviews.

\begin{tabular}{|c|c|c|}
\hline Domains (outcomes) & $\begin{array}{l}\text { No } \text {. of medium quality reviews }(n=8) \\
\text { (references), or high quality }(n=1)\end{array}$ & $\begin{array}{l}\text { No'. of low quality } \\
\text { reviews }(n=16)\end{array}$ \\
\hline Loneliness & $\begin{array}{c}7 \text { (Chen \& Schulz, 2016; Choi, Kong, \& Jung, } \\
\text { 2012; Forsman \& Nordmyr, 2017; Forsman et } \\
\text { al., 2017; Miller et al., 2014; Morris et al., } \\
\text { 2014; Poscia, et al., 2018) }\end{array}$ & 6 \\
\hline Social isolation & $1($ Chen \& Schulz, 2016) & 2 \\
\hline $\begin{array}{l}\text { Social interaction or social } \\
\text { support }\end{array}$ & $\begin{array}{c}3 \text { (Chen \& Schulz, 2016; Forsman \& Nordmyr, } \\
\text { 2017; Morris et al., 2014) }\end{array}$ & 6 \\
\hline $\begin{array}{l}\text { Participation, social } \\
\text { connectedness or social } \\
\text { inclusion }\end{array}$ & $\begin{array}{l}4 \text { (Chen \& Schulz, 2016; Forsman \& Nordmyr, } \\
\text { 2017; Miller et al., 2014; Morris et al., 2014) }\end{array}$ & 2 \\
\hline $\begin{array}{l}\text { Self-efficacy, self-esteem or } \\
\text { empowerment }\end{array}$ & $\begin{array}{c}3 \text { (Forsman \& Nordmyr, 2017; Forsman et al., } \\
\text { 2017; Morris et al., 2014) }\end{array}$ & 1 \\
\hline Depression & $\begin{array}{l}5 \text { (Bleakley et al., 2013; Choi et al., 2012; } \\
\text { Forsman et al., 2017; Morris et al., 2014; } \\
\text { Ronzi, et al., 2018) }\end{array}$ & 9 \\
\hline Cognition & 1 (Bleakley et al., 2013) & 4 \\
\hline $\begin{array}{l}\text { Quality of life (QoL), life } \\
\text { satisfaction or well-being }\end{array}$ & $\begin{array}{c}5 \text { (Bleakley et al., 2013; Forsman et al., 2017; } \\
\text { Miller et al., 2014; Morris et al., 2014; Ronzi, } \\
\text { et al., 2018) }\end{array}$ & 5 \\
\hline $\begin{array}{l}\text { Physical health or physical } \\
\text { activity }\end{array}$ & $\begin{array}{l}4 \text { (Bleakley et al., 2013; Forsman \& Nordmyr, } \\
\text { 2017; Miller et al., 2014; Ronzi, et al., 2018) }\end{array}$ & 5 \\
\hline ADL performance & 0 & 0 \\
\hline Autonomy & 0 & 1 \\
\hline Usability & $\begin{array}{c}3 \text { (Bleakley et al., 2013; Forsman \& Nordmyr, } \\
\text { 2017; Forsman et al., 2017) }\end{array}$ & 3 \\
\hline $\begin{array}{l}\text { Security or risk (mental or } \\
\text { physical) }\end{array}$ & $\begin{array}{c}3 \text { (Bleakley et al., 2013; Forsman \& Nordmyr, } \\
\text { 2017; Miller et al., 2014) }\end{array}$ & 2 \\
\hline
\end{tabular}

${ }^{1}$ Total no. of reviews. Same review relevant for several domains.

The digitalization of society, including health and social care, transforms activities, communication, and interaction between people and the environment (Swedish Government, 2015). Evidence of how to support older adult's mental health by use of digital technology is sparse, but it is indicated that digital technology that is developed and adapted to meet the older adults' needs can support for example independence in daily life (Lindenberger, Lövdén, Schellenbach, Li, \& Krüger, 2008).

As a part of the digitalization, different digital tools and services are increasing, for example, mobile GPS trackers to support independent and safe walks (Werner et al., 2012), and robotics that might support eating or taking a shower independently (Dahl \& Kamel Boulos, 2013). To meet the prerequisites and requests of older adults, the individual perspective needs to be considered and supported when using and developing digital tools (Rodeschini, 2011). Also, the possibility to benefit from digitalization in society is dependent on individual digital competence, i.e., the understanding of and ability to manage dif- ferent digital tools (Swedish Government, 2016). A previous Swedish study concludes that ICT access and literacy in terms of discursive and social resources, influence inclusion in a digitalised society (Olsson, Samuelsson \& Viscovi, 2019).

In summary, it has been noted that older adults need individually targeted support to participate in the digitalized society and to use digital technology/tools. Research indicates that the use of the internet as a digital tool might positively support older adults, and thereby achieve positive health effects (Jinmoo, Sanghee, Sunwoo, Hee, \& Junhyoung, 2015). To further support older adults in using digital tools, it is necessary to describe the knowledge and the potential effects of digitalisation on healthy aging, including mental health by social engagement. In this study, we recognise that engagement in different social activities for enjoyment and by own choice, can contribute to mental health. Therefore, the objective of this study was to systematically map and describe the scientific knowledge and knowledge gaps on the effects of different types of digital tools for outcomes related to social en- 
gagement in older adults at risk for mental health decline. How to perform an evidence map are described differently and lacks a clear definition (Miake-Lye, Hempel, Shanman, \& Shekelle, 2016). In this study, a systematic evidence map has been used to conclude evident knowledge as well as knowledge gaps, based on systematic reviews in the research field. More specifically, the systematic map aimed to provide scientific evidence and identify scientific knowledge gaps in relation to:

- digital tools as a means of promoting social engagement in older adults at risk of mental health decline

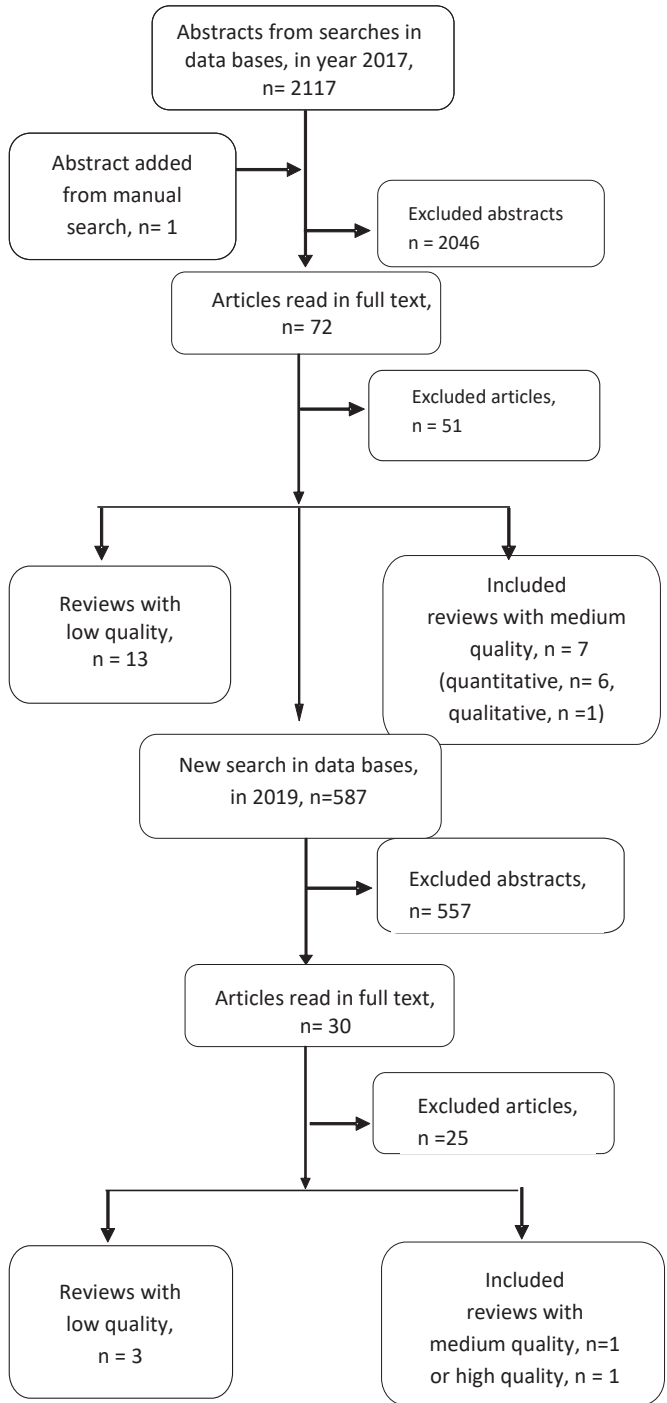

Figure 1. Flowchart describing the total number (n) of included and excluded abstracts, articles read in full text, as well as the number of systematic reviews with low or medium quality included in the evidence map.
- older adults' experiences and/or other outcomes when using digital tools

- studied and described disadvantages, difficulties or risks related to digital tools

\section{Method}

A systematic evidence map was performed based on systematic reviews with quantitative and/or qualitative primary studies.

\section{Domains}

In total, 13 groups of outcomes related to social engagement and the use of digital tools were predefined (they are referred to here as domains). If a scientific knowledge gap was prominent, no systematic reviews relevant to the domain were identified, or there was insufficient evidence to determine the effects of the intervention.

\section{Selection criteria}

Inclusion criteria were defined using PICOS; Population (P); people 60 years of age or older with mental illness or those at risk of mental health decline. Interventions (I); use of digital tools (i.e., computer, mobile phones and software applications) that can be purchased or prescribed by registered healthcare personnel. Comparisons (C); only systematic reviews were included. Outcomes $(\mathrm{O})$, defined domains; Loneliness, social isolation, social interaction, participation, selfefficacy, depression, cognition, Quality of life (QoL), Physical health or physical activity, ADLperformance, autonomy, usability, security or risk. Complete list of domains in Table 1. Study design (S); systematic reviews assessing qualitative or quantitative study results.

Exclusion criteria: studies before 2007 were excluded due to the rapid changes in this research area. Also, studies evaluating, older adults with dementia, experiences of living with mental illness, acceptance and adaptation of technology, e-health, smart homes (including sensors and safety alarms), and medical aids and mobile tools used by social care staff (e.g. care planning tools or medication apps). Lastly, specific treatments (e.g. Cognitive Behavioural Therapy), and education for professionals were also excluded.

\section{Systematic literature search}

A systematic literature search was designed to identify all published systematic reviews potentially relevant to the selection criteria and the 13 pre-defined domains of eligible outcomes. The search was conducted in April 2017 that were updated in September 2019 in Academic Search Elite, Cinahl, Cochrane Library, Psyclnfo, PubMed, Soclndex, Social Services Abstract/ Sociological Abstract, and Scopus (in April 2017 in: IBSS and ASSIA). Furthermore, Epistemonikos, EuroScan, HTA Database, CRD, NICE, 
Table 2. Overview of scientific evidence and evidence gaps, respectively.

\section{Domains (outcomes) Scientific evidence (conclusion from the authors} of the reviews)

Loneliness

\author{
Internet and computer training: Significant \\ decrease in loneliness (Choi et al., 2012) \\ ICT 1 including iPad/smartphones, chats, \\ interactive games/applications, and e-mail: \\ significant positive effect in terms of decreased \\ loneliness (Chen \& Schulz, 2016).
}

Internet use: Future loss of internet as communication alternative experienced as a threat to mental health, due to risk of loneliness (Forsman \& Nordmyr, 2017)*
Evidence gap (review is missing or presents limited support, inconclusive findings, or evidence gap stated by the authors of the reviews)

Internet and computer training: Effect inconclusive, one primary study shows significant effect $(p<0.001)$

(Forsman et al., 2017).

ICT $^{1}$ Primary studies on general internet use $(n=2)$ and social media $(n=1)$ identified negative or non-significant outcome. Related to video calls inconclusive findings (Chen \& Schulz, 2016).

Smart technology incl. interactive solutions and websites (intended to support social contacts): Three out of five studies indicate significant positive findings and the two others insufficient support (Morris et al., 2014).

$V^{2}$ and gaming in home environment: Insufficient support for recommendation (Miller et al., 2014).

ICT ${ }^{1}$ and computer use: primary studies identified significant reduced loneliness after intervention (Poscia, et al., 2018).

Social isolation $\quad$ ICT ${ }^{1}$ including general internet use, support via phone, computer or internet: significant effect on social isolation (Chen \& Schulz, 2016).

ICT ${ }^{1}$ including general internet use, support via phone, computer or internet: Unclear effect on social isolation as an overarching concept (Chen \& Schulz, 2016).

Internet use: Not clear how older people would prefer

social support social support (Chen \& Schulz, 2016).

Smart technology interactive solutions and websites Significant improvement of social support (6 primary studies) (Morris et al., 2014).

Internet use: Older people experience that new, better ways to interact and more frequent communication promotes social interaction (Forsman \& Nordmyr, 2017)*

Participation, social $\quad$ ICT $^{\mathbf{1}}$ : Use of social media, video calls, chat, mobile connectedness or social phone or smartphone, iPads, showed significant inclusion positive effect on belonging (Chen \& Schulz, 2016).

Internet use: Experiences of social inclusion is enabled by ability and knowledge to bridge the "digital gap" to be included in the IT-community (Forsman \& Nordmyr, 2017)*.
to receive support to use the internet (Forsman \& Nordmyr, 2017)

Internet and computer training: More studies needed to show the effect in more vulnerable groups, i.e., family care givers or people in rural settings with limited transportation possibilities (Forsman et al., 2017).

\section{Smart technology incl. interactive solutions and websites (intended to support social contacts):} Relatively few studies have investigated the effects of smart technology on social connectedness. Social connectedness is a multidimensional phenomenon that includes social supports as well as participation, empowerment, engagement, isolation and loneliness further complicating the comparison of outcomes (Morris et al., 2014).

$V^{2}$ and gaming in home environment: Insufficient support regarding the effect of VR/gaming on physical activity to prevent participation restriction (Miller et al., 2014).

Self-efficacy, selfesteem or empowerment

\begin{abstract}
Internet use: Mastering new technology was found to increase mental health by promoting a feeling of competence and empowerment as well as
\end{abstract} increased self-esteem (Forsman \& Nordmyr, 2017)*.
Smart technology incl. interactive solutions and websites: Three out of five studies indicate significant positive effect (empowerment and self -efficacy) [Morris, et. al., 2014].

Internet and computer training: Unclear effect in one primary study (self-esteem) (Forsman et al., 2017).

\begin{tabular}{|c|c|}
\hline \multirow[t]{2}{*}{ Depression } & $\begin{array}{l}\text { Internet and computer training: No significant } \\
\text { difference (Choi et al., 2012). }\end{array}$ \\
\hline & $\begin{array}{l}\text { Internet and computer training: Significant } \\
\text { decrease of depressive symptoms (Forsman et al., } \\
\text { 2017). }\end{array}$ \\
\hline
\end{tabular}

ICG $^{3}$ : Significant positive effects on depressive symptoms according to one primary study (Bleakley et al., 2013)

Smart technology incl. interactive solutions and websites: Inconclusive findings (Morris et al., 2014). ICT $^{\mathbf{1}}$ : A non-significant reduction in depression was detected (Ronzi, et al., 2018).

ICG $^{3}$ : Indicating positive effect on cognition (Bleakley et al., 2013).
OTSeeker, PEDRO, Prospero, and PubMed Health were searched for systematic reviews and HTA-publications. Reference lists of identified systematic reviews, as well as protocols from Campbell and the Cochrane Collaboration, were also searched (The search strategies in Appendix I). 
Table 2 (cont.). Overview of scientific evidence and evidence gaps, respectively.

\section{Domains (outcomes) $\quad$ Scientific evidence (conclusion from the authors of the reviews)}

Evidence gap (review is missing or presents limited support, inconclusive findings, or evidence gap stated by the authors of the reviews)

Quality of life (QoL),

life satisfaction or well-

being

Internet and computer training: Inconclusive effect, one primary study shows decreased QoL $(p<0.01)$ in both control and intervention group, but also increased life satisfaction in the intervention group, but not control group ( $p<0.001$ ) (Forsman et al., 2017).

$V^{2}$ and gaming in home environment: Insufficient support for recommendations related to well-being (Miller et al., 2014).

ICG $^{3}$ : Indicating positive effect on QoL (Bleakley et al., 2013).

Smart technology incl. interactive solutions and websites: Four of five studies show some positive effect on QoL (Morris et al., 2014).

ICT $^{\mathbf{1}}$ was reported to improve experiences of well-being in one qualitative primary study. In the quantitative studies inconclusive results for QoL were reported (Ronzi, et al., 2018).

Physical health or $\quad \mathbf{I C G}^{\mathbf{3}}$ : Positive effect on balance and postural physical activity

$$
\text { control (Bleakley et al., 2013). }
$$

Internet use: Was found to provide extended access to resources in society, enabling a more active life based on personal wishes and needs (Forsman \& Nordmyr, 2017)*.
Usability
VR ${ }^{2}$ and gaming in home environment: Insufficient support for recommendation related to physical activity (Miller et al., 2014).

ICT': including computer use, showed non-significant results on physical and mental health in one primary study (Ronzi, et al., 2018).

ICG $^{3}$ : Insufficient support for conclusive conclusion, except for physical health (Bleakley et al., 2013).

Internet use: Different groups' ICT needs and wishes (Forsman \& Nordmyr, 2017) ${ }^{*}$.

Internet and computer training: Needs, use and outcomes for different groups of older people, e.g., with disability and different socioeconomic backgrounds not known (Forsman et al., 2017).

ICG $^{\mathbf{3}}$ : A minor number of unwanted effects were reported such as muscular inconveniences related to uncommon movements or loading. Most primary studies did not

include such outcomes (Bleakley et al., 2013).

$V^{2}$ and gaming in home environment: Aspects of security such as prevention of falls, and observation of vital signs (Miller et al., 2014).

Information and communication technology; ${ }^{2}$ Virtual reality; ${ }^{3}$ nteractive computer games.

*Findings from qualitative data collection with individual interviews, focus groups or surveys using free text responses.

"In most reviews not an outcome, but rather an aspect discussed.

\section{Analysis}

The relevance of the studies in the final sample was assessed by reading the abstracts and comparing them to the PICOS. Two people, independently of each other, performed the relevance assessment. In April 2017, the scientific quality of the remaining 20 systematic reviews was assessed by the same two people using the 11 items of the AMSTAR manual (Shea et al., 2007). The same procedure was repeated in September 2019, where five systematic reviews was assessed in the same way. In every step, discrepancies were solved by consensus, and secondary opinions were collected if a consensus was not reached. The flow chart with the number of included and excluded articles is presented in Figure 1.

The results from the identified relevant systematic reviews were compiled without any evidence grading or relevance or risk of bias assessment of the primary studies included in the systematic reviews (Description of included reviews in Appendix II. Excluded references in Appendix III).

\section{Results}

In this section, the domains of outcomes are first summarised, followed by a description of findings from the systematic reviews.

\section{Domains of outcomes}

In total, 25 systematic reviews answering the objective of this evidence map were identified. Of these 25 reviews, one was graded as high quality, eight were graded as medium quality (Table 1) and 16 were graded as low quality (Appendix IV).

\section{Findings from the systematic reviews}

Related to the included systematic reviews scientific knowledge was found, as well as knowledge 
gaps (Table 2). The results from the systematic reviews are summarised and described in two sub-headings: Interactive computer games related to physical, mental, or cognitive outcomes and quality of life and Information and communication technology related to psychological and social outcomes and quality of life.

Interactive computer games related to physical, mental or cognitive outcomes and quality of life This section describes the effects of digital tools such as interactive computer games, i.e. Nintendo Wii, virtual reality (VR), or dance mats, on the outcomes of depression, cognition, quality of life, and physical activity that were studied in two systematic reviews (Bleakley et al., 2013; Miller et al., 2014). The number of participants in the primary studies included in these reviews varied from 1 to 60 . Exercise-induced by interactive computer games proved to be more effective than other physical training to promote balance (Bleakley et al., 2013). However, no recommendations regarding the type of exercises, dose, duration, or intensity were given. There is, however, some support that exercise reduces depressive symptoms and promotes QoL. On the other hand, Miller et al. (2014) reported that the existing knowledge base is weak and, therefore, no recommendations can be given regarding the effectiveness of VR/digital games as a means of promoting physical activity or reducing disability, activity limitations and participation restrictions (Miller et al., 2014). Interactive computer games are described as essentially safe (Bleakley et al., 2013). However, a few individuals chose to interrupt physical activity when using dance mats due to pain (Miller et al., 2014). For safety reasons, it is therefore of great importance that these individuals are closely observed when exercising (Miller et al., 2014).

Information and communication technology related to psychological and social outcomes and quality of life

Information and Communication Technology (ICT) is described in seven of the systematic overviews (Chen \& Schulz, 2016; Choi, et al., 2012; Forsman \& Nordmyr, 2017; Forsman et al., 2017; Morris, et al, 2014; Poscia, et al., 2018; Ronzi, et al, 2018). Chen and Schulz show positive effects from using the internet or web-based apps (e.g. search function, e-mail, chat, video conferencing, social media) on social isolation, social support, and social connectedness with other people and surrounding communities (Chen \& Schulz, 2016). ICT can reduce social isolation by promoting contact with the outside world, including friends and family, contributing to social support, and promoting involvement in activities that they found interesting (Chen \& Schulz, 2016). In addition, self-esteem was strengthened as the older adults felt younger and included in the "modern generation", as well as remaining socially active, overcoming challenges and gaining new abilities from the intervention (Chen \& Schulz, 2016). This also had additional positive effects on life satisfaction. However, the effect of using the internet or web-based apps rarely lasted more than six months. Findings relating to loneliness were inconsistent, and the effects on self-esteem and control in life were not-significant (Chen \& Schulz, 2016). In a meta-analysis performed by Choi et al. (2012), computer and internet-based interventions (mainly computer usage and e-mail management), significantly reduced loneliness but were ineffective in reducing depression (Choi, Kong, \& Jung, 2012).

Internet-based programmes for older adults, lasting from four weeks up to three years, including health-related information, self-help interventions, peer support, or communication with healthcare professionals increased social support significantly (Morris et al., 2014). Interactions with others via the internet positively affected empowerment in three out of five primary studies included in the review by Morris et al (2014). Similarly, loneliness was significantly reduced in three out of five studies in the review by Morris et al (2014). Some positive effects were also found for QoL, while the impact on depression and self-esteem was inconsistent. However, a significant positive effect on depressive symptoms was found after training in computer usage (how to use the internet, email, etc.) (Forsman et al., 2017), and significantly influenced either reduced loneliness or increased social contacts (Poscia, 2018). Though many different interventions were combined, and only four used ICT, therefore the results are more preliminary. In Ronzi et al., (2018) non-significant effects on depression were found. However, both reviews by Chen and Schultz (2016) and Morris et al. (2014) conclude that the respective outcomes (i.e. social isolation and social connectedness) are multidimensional phenomena. These phenomena are measured and defined in different ways in the primary studies, which negatively affects the possibility to compare the results between studies (Chen \& Schulz, 2016). Similarly, methodologically strong or coherent evaluations of the effects of computer training and internet use for mental well-being are few (Forsman et al., 2017), which could be because the primary outcome (mental well-being) lacks a clear definition and is, therefore, difficult to study.

The risks, difficulties, or disadvantages of ICT for the individual are rarely included in the reviews (Chen \& Schulz, 2016; Choi et al., 2012; Morris et al., 2014). However, it is found that technology that is not individually adapted may limit or prevent potential positive effects of ICT (Choi et al., 
2012). ICT does not suit all older adults, and individual training and consideration of the interests as well as the motivation of the individual are vital when introducing digital tools (Chen \& Schulz, 2016). Other factors (e.g. socioeconomic conditions, personal interests, or the ability to manage technology) that could prevent or promote the use of computers need to be considered when introducing digital tools, since these, in turn, may influence mental well-being (Forsman et al., 2017).

Forsman and Nordmyr (2017) summarised the results of 14 qualitative studies focusing on older adults' experiences of using the internet (for example for communication) and its impact on psychosocial health (Forsman \& Nordmyr, 2017). Communication (primarily via e-mail) was described as one way of finding new social contacts, as well as increasing, improving, and maintaining existing contacts with friends and family. The more experienced internet users feared they would lose the possibility to communicate via the internet since it might increase experiences of loneliness and in turn negatively impact their mental health. Failing to cope with future technological developments (i.e., being knowledgeable and having updated technique), and thus becoming dependent on others was another identified risk. To achieve the positive benefits of using the internet, sufficient support was considered vital (Forsman \& Nordmyr, 2017). Online groups provided valuable support, especially if the same support was not possible to obtain elsewhere. ICT also made areas for activity and participation (e.g. community service and interest groups) more accessible according to the participants, with additional positive effects on mental health (Forsman \& Nordmyr, 2017).

\section{Discussion}

This evidence map is performed at an early stage of a rapidly developing area. Findings indicate somewhat inconclusive but positive effects, for example, that education about and use of the internet might reduce loneliness. Scientific knowledge gaps were identified in the domains of cognition, quality of life, and usability, and no results for the domains of ADL-performance and autonomy were found. An evidence map, like this one, only displays existing scientific knowledge and knowledge gaps. The degree of evidence is not graded.

Regarding the use of interactive computer games, it is indicated that, for example, balance (Bleakley et al., 2013), QoL, and depression can be successfully influenced by using games. This is promising given the fast development in this particular area potentially bringing new, and hopefully even better games. However, more studies are needed before recommendations of intervention programmes (including dose and inten- sity) can be given. Even though further research is needed to create a solid evidence base and future recommendations, interactive computer games and ICT are in general safe to use. None of the nine systematic reviews reported any harmful effects of using digital technology/tools.

The use of ICT (such as e-mail, video calls, and social media) might influence social isolation positively, as well as social support and social connection to others. Also, experiences of loneliness are indicated to be reduced by the use of computers and the internet (Chen \& Schulz, 2016; Choi et al., 2012; Forsman \& Nordmyr, 2017; Forsman et al., 2017; Morris et al., 2014; Poscia et al., 2018). Similarly, recent research shows that ICT literacy and social networks are important to avoid the risk of digital exclusion (Olsson et al., 2019). It is also known that older adults participate in and use the internet, but the needs and requests when using it vary between individuals and generations (Findahl, 2014). To individually target and adapt, for example, the use of the internet, can be valuable and support usage in older populations. Also, by adapting the technology to expressed needs and preferences, the opportunities to use digital technology/tools to support mental health and social engagement during aging might increase.

The lack of conclusive results in this systematic evidence map can be explained by limited numbers of studies that have evaluated the interventions sufficiently. Moreover, the multitude of concepts used to describe the outcomes (i.e. loneliness, social isolation, social support, social connectedness, or social inclusion) limits the possibilities to generate scientific evidence. The experiences of using digital tools are challenging to evaluate due to the complexity of these interventions. For example, the social environment, support from friends and family, access to technology, and so forth, contribute to this complexity and will influence the effects of the intervention. Such complex interventions are suggested to be evaluated in several steps, and systematic reviews can be the knowledge base to further develop scientific evidence (Craig et al., 2008). Also, to develop the research area, future studies should be well planned when it comes to studying size, design, and quality (Krefting, 1991; Wang \& Bakhai, 2005). Adding to this complexity, it is demanding, yet important, for evaluative research to keep up with this rapid development.

Further, considering quality aspects of the research included in the present evidence map, only one of the included systematic reviews comprised a meta-analysis (Choi, 2012). In the initial search, none of the systematic reviews used an established tool to grade the quality of the evi- 
dence. In the updated search in 2019, both systematic reviews (Poscia, et al, 2018; Ronzi, 2018) used a tool to grade the quality of the included reviews. Also, two of the included reviews evaluated publication bias, which should be considered in all systematic reviews to avoid biased evidence (Joober, Schmitz, Annable, \& Boksa, 2012). All these aspects of stronger research designs should be further considered in future studies to increase and strengthen the knowledge base in this research area. It deserves also to mention that the systematic review graded to be of high quality (Ronzi, et al., 2018), focused on a variety of interventions where only four of the included articles applied digital tools of some sort. Thus, also findings from this high-quality review need to be interpreted with caution.

Context is also important to consider in an evidence map, and the primary studies in the overviews were completed in different parts of the Western world. This reflects international interest in the research area but may limit transferability to a local context. This is relevant since the organisation of social care differs between countries, as well as the availability and funding of different digital technology/tools for individuals. Finally, in terms of the study population, systematic reviews targeted older adults. The majority of the participants were 60 years old or older, and a few were 45 years old or older (Chen \& Schulz, 2016; Miller et al., 2014; Morris et al., 2014; Pos- cia, et al, 2018). The primary focus of this map was not to include only older adults diagnosed with mental illness, but instead to use a health promotion or holistic perspective. The systematic reviews included different target groups such as older adults at risk of experiencing loneliness (Choi et al., 2012) or with limited physical abilities (Bleakley et al., 2013). Older adults that had experienced a stroke (Chen \& Schulz, 2016) or suffered from other illnesses were also included as well as healthy individuals in some of the studies. This variety of older adults in the reviews can be considered as representative of a normal, heterogenic population of older adults. Nevertheless, Forsman and Nordmyr (2017) conclude that important subgroups reflecting socioeconomic positions or more vulnerable groups are missing in their included studies, and this is likely to be true for several of the included reviews. The possibilities to generalise the results to these groups or older adults solely with mental illness might, therefore, be limited.

\section{Conclusion}

The systematic map shows early and somewhat inconclusive evidence in a developing research area. Future research should include larger, controlled studies, also representing various subgroups of older adults in different contexts, which evaluate the effects of using digital tools to promote healthy aging of older adults and support social engagement.

\section{Acknowledgements}

The information search was done by Agneta Brolund, Information specialist at Swedish Agency for Health Technology Assessment and Assessment of Social Services (SBU), Stockholm, Sweden; the evidence map is funded by Swedish Agency for Health Technology Assessment and Assessment of Social Services (SBU), Stockholm, Sweden.

\section{References}

Bleakley, C. M., Charles, D., Porter-Armstrong, A., McNeill, M. D. J., McDonough, S. M., \& McCormack, B. (2013). Gaming for Health: A Systematic Review of the Physical and Cognitive Effects of Interactive Computer Games in Older Adults. Journal of Applied Gerontology, 34(3), NP166-NP189. https:// doi.org/10.1177/0733464812470747

Chen, Y., \& Schulz, P. (2016). The Effect of Information Communication Technology Interventions on Reducing Social Isolation in the Elderly: A Systematic Review. Journal of Medical Internet Research., 18(1). https://doi.org/10.2196/jmir.4596

Choi, M., Kong, S., \& Jung, D. (2012). Computer and Internet Interventions for Loneliness and Depression in Older Adults: A Meta-Analysis. Healthcare Informatics Research, 18(3), 191-198. https://doi. org/10.4258/hir.2012.18.3.191

Craig, P., Dieppe, P., Macintyre, S., Michie, S., Nazareth, I., \& Petticrew, M. (2008). Developing and Evaluat- ing Complex Interventions: The New Medical Research Council Guidance. BMJ, 337(7676). https:// doi.org/10.1136/bmj.a1655

Dahl, T., S., \& Kamel Boulos, M., N. (2013). Robots in Health and Social Care: A Complementary Technology to Home Care and Telehealthcare? Robotics, 3(1), 1-21. https://doi.org/10.3390/robotics3010001

Findahl, O. (2014). Pensionärerna och Internet [Retirees and the Internet]. Stockholm: .SE. Retrieved from: https://internetstiftelsen.se/docs/Pensionarerna_och_internet_2014.pdf

Forsman, A. K., \& Nordmyr, J. (2017). Psychosocial Links Between Internet Use and Mental Health in Later Life: A Systematic Review of Quantitative and Qualitative Evidence. Journal of Applied Gerontology, 36(12), 1471-1518. https://doi. org/10.1177/0733464815595509

Forsman, A. K., Nordmyr, J., Matosevic, T., Park, A. L., Wahlbeck, K., \& McDaid, D. (2017). Promoting Mental Wellbeing Among Older People: Technology-Based Interventions. Health Promotion International, 33 (6), 1042-1054. https://doi.org/10.1093/ heapro/dax047

Jinmoo, H., Sanghee, C., Sunwoo, L., Hee, L. K., \& Junhyoung, K. (2015). Internet Use and Well-Being in Older Adults. Cyberpsychology, Behavior, and Social Networking, 18(5), 268-272. https://doi. 
org/10.1089/cyber.2014.0549

Joober, R., Schmitz, N., Annable, L., \& Boksa, P. (2012). Publication Bias: What Are the Challenges and Can They be Overcome? Journal of Psychiatry and Neuroscience, 37(3), 149-152

Krefting, L. (1991). Rigor in Qualitative Research: The Assessment of Trustworthiness. The American Journal of Occupational Therapy: Official Publication of the American Occupational Therapy Association, 45(3), 214-222. https://doi.org/10.5014/ ajot.45.3.214

Lindenberger, U., Lövdén, M., Schellenbach, M., Li, S.C., \& Krüger, A. (2008). Psychological Principles of Succesful Aging Technologies: A Mini-Review. Gerontology, 54(1), 59-68.

Miake-Lye, I. M., Hempel, S., Shanman, R., \& Shekelle P. G. (2016). What is an evidence map? A systematic review of published evidence maps and their definitions, methods, and products. Systematic reviews, 5 (28), 1-21. https://doi.org/10.1186/s13643016-0204-x

Miller, K. J., Adair, B. S., Pearce, A. J., Said, C. M., Ozanne, E., \& Morris, M. M. (2014). Effectiveness and Feasibility of Virtual Reality and Gaming System Use at Home by Older Adults for Enabling Physical Activity to Improve Health-Related Domains: A Systematic Review. Age and Ageing, 43(2), 188-195. https://doi.org/10.1093/ageing/aft194

Morris, M. E., Adair, B., Ozanne, E., Kurowski, W., Miller, K. J., Pearce, A. J., Said, C. M. (2014). Smart Technologies to Enhance Social Connectedness in Older People who live at Home. Australian Journal on Ageing, 33(3), 142-152. https://doi.org/10.1111/ ajag. 12154

Poscia, A., Stojanovic, J., Ignazio La Milia, D., Duplaga, M., Grysztar, M., Moscate, U., Mahnavita, N. (2018). Interventions targeting loneliness and social isolation among the older people: An update systematic review. Experimental Gerontology, 102 (February), 133-144. https://doi.org/10.1016/j. exger.2017.11.017

Rodeschini, G. (2011). Gerotechnology: A new kind of care for aging? An Analysis of the Relationship Between Older People and Technology. Nursing and Health Sciences, 13(4), 521-528.

Ronzi, S., Orton, L., Pope, D., Valtorta, N, K., Bruce, N, G. (2018). What is the impact on health and wellbeing of interventions that foster respect and so- cial inclusion in community residing older adults? A systematic review of quantitative and qualitative studies. Systematic reviews, 7 (26), 1-22. https://doi. org/10.1186/s13643-018-0680-2

Shea, B. J., Grimshaw, J. M., Wells, G. A., Boers, M., Andersson, N., Hamel, C., Bouter, L. M. (2007). Development of AMSTAR: a measurement tool to assess the methodological quality of systematic reviews. BMC medical research methodology, 7(1), 10. https://doi.org/10.1186/1471-2288-7-10

Swedish Government. (2015). Gör Sverige i framtiden - digital kompetens. Delbetänkande av Digitaliseringskommissionen. SOU 2015:28. Stockholm.

Swedish Government. (2016). Slutbetänkande av Digitaliseringskommissionen. SOU 2016:89 För digitalisering i tiden. Stockholm.

The Swedish National Institute of Public Health. (2007). Healthy Ageing - A Challenge for Europe (2007:01). Stockholm, Sweden. Retrieved from: http://www.healthyageing.eu/sites/www.healthyageing.eu/files/resources/S2007_01_Healthy_ageing_eng0706(1).pdf

Olsson, T., Samuelsson, U., Viscovi, D. (2019). At risk of exclusion? Degrees of ICT Access and Literacy among Senior Citizens. Information, Communication and Society, 22(1), 55-72. https://doi.org/10.10 80/1369118X.2017.1355007

Vaughan, L., Corbin, A. L., \& Goveas, J. S. (2015). Depression and frailty in later life: a systematic review. Clinical Interventions In Aging, 10, 1947-1958. https://doi.org/10.2147/CIA.S69632

Wang, D., \& Bakhai, A. (Eds.). (2005 ). Clinical trial: A practical guide to design, analysis and re-porting. London: Remedica.

Werner, S., Auslander, G. K., Shoval, N., Gitlitz, T., Landau, R., \& Heinik, J. (2012). Caregiving burden and Out-of-Home Mobility of Cognitively Impaired Care-recipients based on GPS tracking. 24(11), 1836-1845. https://doi.org/10.1017/ S1041610212001135

World Health Organization (WHO) (2004). Promoting mental health: concepts, emerging evidence, practice : summary report. World Health Organization, Department of Mental Health and Substance Abuse in collaboration with the Victorian Health Promotion Foundation (VicHealth) and the University of Melbourne. Retrived from: https://www.who.int/ mental_health/evidence/en/promoting_mhh.pdf 
- $\quad$ (DE "OLDER people -- Social networks" OR DE "OLDER people" OR DE "FRAIL elderly" OR DE "OLDER men" OR DE "OLDER women" OR DE "RURAL elderly" OR DE "SELF-help devices for older people" OR DE "WEBSITES for older people" OR DE "AGING" OR DE "OLD age" OR DE "RETIREES" OR DE "RETIREMENT") OR TI(old* OR elder* OR aged* OR aging* OR ageing* OR adult* OR senior* OR retire*) OR KW(old* OR elder* OR aged* OR aging* OR ageing* OR adult* OR senior* OR retire*) OR AB(elder* OR aging* OR ageing* OR (old* W2 patient*) OR (old*W2 age*) OR (old* W2 people*) OR senior* OR retirement*)

\section{Intervention: Social robots}

- DE "ASSISTIVE computer technology" OR TI((companion N3 robot*) OR (social* N3 robot*) OR (sociable 38967 N2 robot*) OR (domestic W2 robot*) OR (healthcare N3 robot*) OR Paro OR (video W2 game*) OR (assistive W2 technolog*) OR gerontechnology) OR AB((companion N3 robot*) OR (social* N3 robot*) OR (sociable N2 robot*) OR (domestic W2 robot*) OR (healthcare N3 robot*) OR Paro OR (video W2 game*) OR (virtual W2 realit*) OR (virtual W2 environment*) OR "science and technology studies" OR (assistive W2 technolog*) OR gerontechnology)

\section{Intervention: ICT/Social media}

- DE "INFORMATION technology" OR DE "SOCIAL informatics" OR DE "TELECOMMUNICATION" OR DE 306614 "TELEPRESENCE" OR DE "INTERNET" OR DE "INTERNET \& older people" OR DE "INTERNET entertainment" OR DE "TELEVISION \& older people" OR DE "SELF-help devices for older people" OR DE "WEBSITES for older people" OR DE "SOCIAL media" OR DE "BLOGS" OR DE "COMPUTER bulletin boards" OR DE "ONLINE chat" OR DE "ONLINE social networks" OR DE "PODCASTS" OR DE "WIKIS (Computer science)" OR DE "VIRTUAL communications" OR DE "VIRTUAL work teams" OR DE "VIRTUAL reality" OR DE "SHARED virtual environments" OR DE "WORLD Wide Web" OR DE "CELL phones" OR DE "TELEPHONE" OR DE "SMARTPHONES" OR DE "MOBILE television" OR DE "PERSONAL communication service systems" OR DE "TEXT messages (Telephone systems)" OR DE "EMAIL" OR DE "ELECTRONIC discussion groups" OR DE "FORUMS (Discussion \& debate)" OR DE "ELECTRONIC games" OR DE "TELECOMMUNICATION in medicine"

- $\quad$ TI ((information N3 technol*) OR ICT OR "science and technology studies" OR (virtual W2 realit*) OR (virtual W2 environment*) OR internet* OR computer* OR digital OR online OR (web w2 based*) OR (web W2 2.0) OR (social W2 networking*) OR twitter* OR facebook* OR (face W1 book*) OR blogging OR blogs OR (social W2 media*) OR (electronic W2 mail*) OR "e-mail" OR email* OR (text W2 messag*) OR sms OR mms* OR phone* OR telephone* OR android* OR iphone* OR smartphone* OR cellphone* OR mobile* OR skype OR apps* OR ipad* OR multimedia* OR (multi W2 media*) OR technolog* OR video*) OR KW((information N3 technol*) OR ICT OR "science and technology studies" OR (virtual W2 realit*) OR (virtual W2 environment*) OR internet* OR computer* OR digital OR online OR (web w2 based*) OR (web W2 2.0) OR (social W2 networking*) OR twitter* OR facebook* OR (face W1 book*) OR blogging OR blogs OR (social W2 media*) OR (electronic W2 mail*) OR "e-mail" OR email* OR (text W2 messag*) OR sms OR mms* OR phone* OR telephone* OR android* OR iphone* OR smartphone* OR cellphone* OR mobile* OR skype OR apps* OR ipad* OR multimedia* OR (multi W2 media*) OR technolog* OR video* OR ehealth) OR AB ((information N3 technol*) OR "science and technology studies" OR (virtual W2 realit*) OR ((virtual W2 environment*) OR digital OR (web w2 based*) OR (web W2 2.0) OR (social W2 networking*) OR twitter* OR facebook* OR (face W1 book*) OR (social W2 media*) OR (electronic W2 mail*) OR "e-mail" OR email* OR (text W2 messag*) OR sms OR mms* OR phone* OR telephone* OR android* OR iphone* OR smartphone* OR cellphone* OR mobilephone* OR skype OR apps* OR ipad* OR multimedia* OR (multi W2 media*) OR technolog* OR (video W2 game*) OR ehealth)

\section{Mental health/Social isolation/Social interaction}

MH "Social Participation" OR DE "SOCIAL networks" OR MH "Social Isolation" OR MH "Social

Alienation" OR MH "Loneliness" OR MH "Risk for Loneliness (NANDA)" OR DE "SOCIAL interaction" OR DE "DEPRIVATION (Psychology)" OR DE "EMOTIONAL deprivation" OR DE "WELL-being" OR DE "JOY" OR DE "MENTAL health" OR DE "ACTIVE aging" OR DE "HAPPINESS in old age" OR DE "SOCIAL role" OR DE "GEROPSYCHOLOGY" OR DE "ANXIETY in old age" OR DE "CREATIVE ability in old age" OR DE "DEPENDENCY (Psychology) in old age" OR DE "DISTRESS (Psychology) in old age" OR "IDENTITY (Psychology) in old age" OR DE "LONELINESS in old age" OR DE "LOVE in old age" OR DE "OPTIMISM in older people" OR DE "SELF-efficacy in old age" OR DE "SELF-esteem in old age" OR DE "SELF-perception in old age" OR DE "SELF-realization in old age" OR DE "SELF-reliance in old age" OR DE "SOCIAL participation" OR DE "OLDER people -- Social networks" OR DE "ONLINE social networks" OR DE "SOCIAL support" OR DE "ENCOURAGEMENT" OR DE "SOCIAL dating" OR DE "OLDER people -Recreation" OR DE "POSITIVE psychology" OR DE "SATISFACTION" OR DE "CONTENTMENT" OR DE "DISCONTENT" OR DE "FRUSTRATION" OR DE "HAPPINESS"

- Tl(particip* OR companion* OR leisure OR psychosocial OR empower* OR (social W2 activit*) OR (social W2 behavior*) OR (social W2 activit*) OR (social W2 connect*) OR (social W2 engagement*) OR (social W2 involvement*) OR (social W2 inclusion*) OR (social W2 contact*) OR interact* OR "quality of life" OR (life N2 satisfaction*) OR wellbeing OR relax* OR happiness*OR (positive W2 emotion*) OR (social W2 identit*) OR "self efficacy" OR salutogen* OR (health W2 promotion) OR (health W2 prevention*) OR (illness W2 prevention) OR meaning OR distress* OR mood* OR loneliness OR boredom OR isolation* OR depress* OR anxiety) OR KW(particip* OR companion* OR leisure OR psychosocial OR empower* OR (social W2 activit*) OR (social W2 behavior*) OR (social W2 activit*) OR (social W2 connect*) OR (social W2 engagement*) OR (social W2 involvement*) OR (social W2 inclusion*) OR 
(social W2 contact*) OR interact* OR "quality of life" OR (life N2 satisfaction*) OR wellbeing OR relax* OR happiness*OR (positive W2 emotion*) OR (social W2 identit*) OR "self efficacy" OR salutogen* OR (health W2 promotion) OR (health W2 prevention*) OR (illness W2 prevention) OR meaning OR distress* OR mood* OR loneliness OR boredom OR isolation*OR depress* OR anxiety) OR AB((psychosocial W2 intervent*) OR (social W2 activit*) OR (social W2 connect*) OR (social W2 behavior*) OR (social W2 connection*) OR (social W2 contact*) OR (social W2 interaction*) OR loneliness OR boredom OR isolation* OR "quality of life" OR (life N2 satisfaction*) OR wellbeing OR relax* OR happiness*OR (positive W2 emotion*) OR (social W2 identit*) OR "self efficacy" OR salutogen*OR (health W2 promotion*) OR (health W2 prevention*) OR (illness W2 prevention) OR distress* OR depress* OR anxiety)

$$
\text { - } 6 \text { OR } 7
$$

\section{Systematic reviews, meta-analysis, meta-synthesis}

• $\quad$ (DE "SYSTEMATIC reviews (Medical research)" OR DE "META-analysis" OR DE "META-synthesis) OR TX 97006 ((systematic*N3 review*) OR (metaanaly* OR (meta W2 analy*) OR (technology W2 overview*) OR (technology W2 appraisal*) OR (technology W2 assessment*)) OR TX ((overview* N2 (review* OR systematic OR synthes* OR summary OR umbrella)) OR TX((integrative W3 review) OR (information W2 synthes*) OR (data W2 synthes*) OR (data N2 extract*)) OR JN ("Cochrane Database of Systematic Reviews") OR TX ((systematic W2 synthes*) OR (meta W2 synthes*) OR metasyntheses* OR (meta W2 ethnograph* OR metaethnograph* OR (thematic W2 synthes*) OR "meta-study" OR "meta-summary" OR "critical interpretive syntheses" OR "qualitative cross-case analysis" OR "grounded theory synthesis" OR "meta-interpretation"))

\begin{tabular}{ccc}
\hline Limits & & \\
\hline$\cdot \quad$ Limiters - Language: English, Norwegian & \\
\hline Combined sets & 1373 \\
\hline 1 AND 2 & $\mathbf{5 0}$ \\
\hline 11 AND 9 & 11315 \\
\hline 1 AND 5 AND 8 & $\mathbf{2 4 1}$ \\
\hline 13 AND 9 AND 10
\end{tabular}

The search result, usually found at the end of the documentation, forms the list of abstracts.

$\mathrm{AB}=$ Abstract

$\mathrm{AU}=$ Author

$\mathrm{DE}=$ Term from the thesaurus

$\mathrm{TI}=$ Title

TX = All Text. Performs a keyword search of all the ispeidatabase's searchable fields

ZC = Methodology Index

$*$ Truncation

" " = Citation Marks; searches for an exact phrase

ASSIA via Proquest 18 April 2017

\section{Search terms}

Population: Age

1. SU.EXACT("Brain damaged elderly people" OR "Centenarians" OR "Disabled elderly people" OR

Items found

"Divorced elderly people" OR "Elderly people" OR "Emotionally disturbed elderly people" OR "Gifted elderly people" OR "Hearing impaired elderly people" OR "Homeless elderly people" OR "Housebound elderly people" OR "Language disordered elderly people" OR "Learning disabled elderly people" OR "Low income elderly people" OR "Mentally ill elderly people" OR "Remarried elderly people" OR "Retired people" OR "Sick elderly people" OR "Single elderly people" OR "Terminally ill elderly people" OR "Visually impaired elderly people" OR "Humanistic gerontology" OR "Gerontology" OR "Ageing" OR "Developmentally disabled older people" OR "Disabled older people" OR "Homeless older people" OR "Learning disabled older people" OR "Mentally ill older people" OR "Older people" OR "Unemployed older people" OR "Senior" OR "Seniority")

2. $\quad$ ti(old* OR elder* OR aged* OR aging* OR ageing* OR (old PRE/1 patient*) OR (old PRE/1 age*) OR (old* 40246 PRE/1 people*) OR (older PRE/1 patient*) OR adult* OR senior* OR retire*) OR if(old* OR elder* OR aged* OR aging* OR ageing* OR (old PRE/1 patient*) OR (old PRE/1 age*) OR (old* PRE/1 people*) OR (older PRE/1 patient*) OR adult* OR senior* OR retire*)

3. 1 OR 2

\section{Intervention: Social robots}

4. SU.EXACT("Technical aids") OR ti((companion N/3 robot*) OR (social* N/3 robot*) OR (sociable N/3 robot*) OR (domestic PRE/2 robot*) OR (healthcare N/3 robot*) OR Paro OR (video PRE/2 game*) OR (assistive PRE/2 technolog*)) OR if((companion N/3 robot*) OR (social* N/3 robot*) OR (sociable N/3 robot*) OR (domestic PRE/2 robot*) OR (healthcare N/3 robot*) OR Paro OR (video PRE/2 game*) OR (virtual PRE/2 realit*) OR (virtual PRE/2 environment*) OR "science and technology studies" OR (assistive PRE/2 technolog*)) OR ab((companion N/3 robot*) OR (social*N/3 robot*) OR (sociable N/3 robot*) OR (domestic PRE/2 robot*) OR (healthcare N/3 robot*) OR Paro OR (video PRE/2 game*) OR (virtual PRE/2 realit*) OR (virtual PRE/2 environment*) OR "science and technology studies" OR (assistive PRE/2 technolog*))

\section{Intervention: ICT/Social media}

5. SU.EXACT("Information Technology" OR "Internet" OR "Chat rooms" OR "Telecommunications" OR "Telephones" OR "Mobile phones" OR "Telecommuting" OR "Hypermedia" OR "Electronic mail systems" OR "Telematics" OR "Virtual reality" OR "Interactive video systems" OR "Videogames" OR "Digital" OR "Listserves") 
ti((information N/2 technol*) OR ICT OR "science and technology studies" OR (virtual PRE/2 realit*) OR (virtual PRE/2 environment*) OR internet* OR computer* OR digital OR online OR (web PRE/2 based*) OR (web PRE/2 2.0) OR (social PRE/2 network*) OR twitter* OR facebook* OR "face book" OR blogging OR blogs OR (social PRE/2 media*) OR (electronic PRE/2 mail*) OR "e-mail" OR email* OR (text PRE/2 messag*) OR sms OR mms* OR phone* OR telephone* OR android* OR iphone* OR smartphone* OR cellphone* OR mobile* OR skype OR apps* OR ipad* OR multimedia* OR (multi PRE/2 media*) OR technolog* OR video* OR digital*) OR if((information N/2 technol*) OR "science and technology studies" OR (virtual PRE/2 realit*) OR ((virtual PRE/2 environment*) OR internet* OR computer* OR digital OR online OR (web PRE/2 based*) OR (web PRE/2 2.0) OR (social PRE/2 network*) OR twitter* OR facebook* OR "face book" OR blogging OR blogs OR (social PRE/2 media*) OR (electronic PRE/2 mail*) OR "e-mail" OR email* OR (text PRE/2 messag*) OR sms OR mms* OR phone* OR telephone* OR android* OR iphone* OR smartphone* OR cellphone* OR mobile* OR skype OR apps* OR ipad* OR multimedia* OR (multi PRE/2 media*) OR technolog* OR video*)) OR ab("science and technology studies" OR (virtual $\mathrm{PRE} / 2$ realit*) OR (virtual PRE/2 environment*) OR internet* OR computer* OR digital OR online OR web based* OR (social PRE/2 networking*) OR twitter* OR facebook* OR "face book" OR blogging OR blogs OR (social PRE/2 media*) OR (electronic PRE/2 mail*) OR "e-mail" OR email* OR (text PRE/2 messag*) OR sms OR mms* OR phone* OR telephone* OR android* OR iphone* OR smartphone* OR cellphone* OR (mobile PRE/2 phone*) OR skype OR apps* OR ipad* OR multimedia* OR (multi PRE/2 media*) OR (video PRE/2 game*))

7. 5 OR 6

Mental health/Social isolation/Social interaction

8. SU.EXACT("Isolation") OR SU.EXACT("Loneliness") OR SU.EXACT ("Social networks") OR SU.EXACT 37395 ("Alienation") OR SU.EXACT ("Seclusion") OR SU.EXACT("Social participation") OR SU.EXACT ("Social interaction") OR SU.EXACT("Social activities") OR SU.EXACT("Wellbeing") OR SU.EXACT ("Social wellbeing") OR SU.EXACT ("Psychological wellbeing") OR SU.EXACT ("Connectedness") OR SU.EXACT ("Life satisfaction") OR SU.EXACT ("Quality of life") OR SU.EXACT ("Depression") OR SU.EXACT("Depressed") OR "Anxiety" OR "Social anxiety" OR SU.EXACT("Mental health promotion") OR SU.EXACT("Efficacy" OR "Personal efficacy")

9. $\quad \mathrm{ti}($ leisure OR companion* OR empower OR (psychosocial PRE/2 intervent*) OR (pro PRE/2 social) OR (social PRE/2 activit*) OR (social PRE/2 behavior*) OR (social PRE/2 connect*) OR (social PRE/2 contact*) OR (social PRE/2 interaction*) OR (social PRE/2 engagement*) OR (social PRE/2 involvement*) OR (social PRE/2 inclusion*) OR (social PRE/2 identit*) OR efficacy OR particip* OR loneliness OR boredom OR isolation* OR "quality of life" OR (life N/2 satisfaction*) OR wellbeing OR relax* OR happiness* OR (positive PRE/2 emotion*) OR meaning OR distress* OR mood* OR depress* OR anxiety OR salutogen* OR "health promotion" OR (health PRE/2 prevention*) OR (illness PRE/2 prevention)) OR if(leisure OR companion* OR empower OR (psychosocial PRE/2 intervent*) OR (pro PRE/2 social) OR (social PRE/2 activit*) OR (social PRE/2 behavior*) OR (social PRE/2 connectt*) OR (social PRE/2 contact*) OR (social PRE/2 interaction*) OR (social PRE/2 engagement*) OR (social PRE/2 involvement*) OR (social PRE/2 inclusion*) OR (social PRE/2 identit*) OR efficacy OR particip* OR loneliness OR boredom OR isolation* OR "quality of life" OR (life N/2 satisfaction*) OR wellbeing OR relax* OR happiness*OR (positive PRE/2 emotion*) OR meaning OR distress* OR mood* OR depress* OR anxiety OR salutogen* OR "health promotion" OR (health PRE/2 prevention*) OR (illness PRE/2 prevention))OR ab((psychosocial PRE/2 intervent*) OR companion* OR (social PRE/2 activit*) OR (social PRE/2 behavior*) OR (social PRE/2 connect*) OR (social PRE/2 contact*) OR (social PRE/2 interaction*) OR (social PRE/2 engagement*) OR (social PRE/2 involvement*) OR (social PRE/2 inclusion*) OR (social PRE/2 identit*) OR efficacy OR particip* OR loneliness OR boredom OR isolation* OR "quality of life" OR (life N/2 satisfaction*) OR wellbeing OR relax* OR happiness*OR (positive PRE/2 emotion*) OR distress* OR depress* OR anxiety OR salutogen* OR "health promotion" OR (health PRE/2 prevention*) OR (illness PRE/2 prevention))

10. 8 OR 9

Study types: Systematic reviews, meta-analysis, meta-synthesis

11. (SU.EXACT("Systematic reviews" OR "Meta-analysis") OR ti((systematic* N/3 review*) OR metaanaly* OR 8183 (meta PRE/2 analy*) OR (technology PRE/2 overview*) OR (technology PRE/2 appraisal*) OR (technology PRE/2 assessment*) OR (overview*N/2 (review* OR systematic OR synthes* OR summary OR umbrella)) OR (integrative PRE/2 review) OR (information PRE/2 synthes*) OR (data PRE/2 synthes*) OR (data N/2 extract*) OR (systematic PRE/2 synthes*) OR (meta PRE/2 synthes*) OR metasyntheses* OR (meta PRE/2 ethnograph*) OR metaethnograph* OR (thematic PRE/2 synthes*) OR "meta-study" OR "meta-summary" OR "critical interpretive syntheses" OR "qualitative cross-case analysis" OR "grounded theory synthesis" OR "meta-interpretation") OR if((systematic*N/3 review*) OR metaanaly* OR (meta PRE/2 analy*) OR (technology PRE/2 overview*) OR (technology PRE/2 appraisal*) OR (technology PRE/2 assessment*) OR (overview* N/2 (review* OR systematic OR synthes* OR summary OR umbrella)) OR (integrative PRE/2 review) OR (information PRE/2 synthes*) OR (data PRE/2 synthes*) OR (data N/2 extract*) OR (systematic PRE/2 synthes*) OR (meta PRE/2 synthes*) OR metasyntheses* OR (meta PRE/2 ethnograph*) OR metaethnograph* OR (thematic PRE/2 synthes*) OR "meta-study" OR "meta-summary" OR "critical interpretive syntheses" OR "qualitative cross-case analysis" OR "grounded theory synthesis" OR "metainterpretation") OR ab ((systematic* N/3 review*) OR metaanaly* OR (meta PRE/2 analy*) OR (technology PRE/2 overview*) OR (technology PRE/2 appraisal*) OR (technology PRE/2 assessment*) OR (overview* N/2 (review* OR systematic OR synthes* OR summary OR umbrella)) OR (integrative PRE/2 review) OR (information PRE/2 synthes*) OR (data PRE/2 synthes*) OR (data N/2 extract*) OR (systematic PRE/2 synthes*) OR (meta PRE/2 synthes*) OR metasyntheses* OR (meta PRE/2 ethnograph*) OR metaethnograph* OR (thematic PRE/2 synthes*) OR "meta-study" OR "meta-summary" OR "critical interpretive syntheses" OR "qualitative cross-case analysis" OR "grounded theory synthesis" OR "metainterpretation")) 
The search result, usually found at the end of the documentation, forms the list of abstracts.

\section{$\mathrm{AB}=$ Abstract}

$\mathrm{AU}=$ Author

$\mathrm{DE}=$ Term from the thesaurus

$\mathrm{TI}=$ Title

TX $=$ All Text. Performs a keyword search of all the sispidatabase's searchable fields

ZC = Methodology Index

$*$ Truncation

" " = Citation Marks; searches for an exact phrase

\section{Cinahl via EBSCO 3 September 2019}

\section{Search terms}

Population: Age

1. MH "Aging" OR MH "Attitude to Aging" OR MH "Aged+" OR MH "Aged, 80 and Over" OR MH "Aged, Hospitalized" OR MH "Frail Elderly" OR MH "Senior Centers" OR MH "Housing for the Elderly" OR (ZG "aged, 80 \& over") or (ZG "aged: $65+$ years")

2. $\quad$ TI(old* OR elder* OR aged* OR aging* OR ageing* OR adult* OR senior* OR retire*) OR AB(old* W2 age*) OR (middle W2 age*) OR middleage* OR (old* W2 patient*) OR (old* W2 people*) OR (old* W2 person*) OR elder* OR aged OR aging OR ageing* OR senior* OR retirement* OR "late life depression" )

3. 1 OR 2

\section{Population: Mental health}

4. $\quad \mathrm{MH}$ "Depression" OR MH "Dysthymic Disorder" OR MH "Depression, Reactive" OR MH "Seasonal Affective Disorder" OR MH "Anxiety" OR MH "Mental Health" OR MH "Depression" OR MH "Social Isolation+" OR MH "Loneliness"

5. TI(depress* OR (mental N3 (problem*OR disorder* OR health*)) OR (dysthymic W2 disorder*) OR anxiet* 109635 OR (social W2 isolation*) OR loneliness OR geropsychology)

6. $\quad 4$ OR 5

\section{Intervention: Social robots}

7. $\quad$ MH "Assisted Living" OR TI((companion N3 robot*) OR (social* N3 robot*) OR (sociable N2 robot*) OR 6428 (domestic W2 robot*) OR (healthcare N3 robot*) OR Paro OR (video W2 game*) OR (assistive W2 technolog*) OR gerontechnology) OR AB((companion N3 robot*) OR (social* N3 robot*) OR (sociable N2 robot*) OR (domestic W2 robot*) OR (healthcare N3 robot*) OR Paro OR "science and technology studies" OR (assistive W2 technolog*) OR gerontechnology)

\section{Intervention: ICT/Social media}

8. $\quad$ MH "Virtual Reality" OR MH "Information Technology" OR MH "Internet" OR MH "Social Media" OR MH 261300 "World Wide Web" OR MH "World Wide Web Applications" OR MH "Telecommunications+" OR MH "Telephone" OR MH "Interactive Voice Response Systems" OR MH "Cellular Phone" OR MH "Text Messaging" OR MH "Instant Messaging" OR MH "Smartphone" OR MH "Computers, Portable" OR MH "Computers, Hand-Held" OR MH "Electronic Bulletin Boards" OR MH "Electronic Mail" OR MH "Audiovisuals" OR MH "Audiorecording" OR MH "Bulletin Boards" OR MH "Television" OR MH "Videorecording" OR MH "Video Games" OR MH "Telehealth"

9. TI ((information N3 technol*) OR ICT OR (virtual W2 realit*) OR (virtual W2 environment*) OR internet* 288079 OR computer* OR digital OR online OR (web W2 based*) OR (web W2 2.0) OR (social W2 network*) OR "social network site" OR twitter* OR facebook* OR (face W1 book*) OR blogging OR blogs OR (social W2 media*) OR (electronic W2 mail*) OR "e-mail*" OR email* OR (text W2 messag*) OR sms OR mms* OR phone* OR telephone* OR android* OR iphone* OR smartphone* OR cellphone* OR mobile* OR skype OR apps* OR ipad* OR multimedia* OR (multi W2 media*) OR technolog* OR video* OR game OR gaming OR digital) OR ehealth) OR AB ((information W2 technol*) OR "science and technology studies" OR (virtual W2 realit*) OR (virtual W2 environment*) OR internet* OR computer* OR digital OR online OR (web W2 based*) OR (web W2 2.0) OR (social W2 network*) OR "social network site" OR twitter* OR facebook* OR (face W1 book*) OR blogging OR blogs OR (social W2 media*) OR (electronic W2 mail*) OR "e-mail*" OR email* OR (text W2 messag*) OR phone* OR telephone* OR android* OR iphone* OR smartphone* OR cellphone* OR (mobile W2 phone*) OR skype OR apps* OR ipad* OR multimedia* OR (multi W2 media*) OR (video W2 game*) OR ehealth)

10. 8 OR 9

\section{Social isolation/Social interaction}

11. MH "Depression" OR MH "Anxiety" OR MH "Social Participation" OR MH "Social Skills" OR MH "Social 365885 Isolation" OR MH "Loneliness" OR MH "Risk for Loneliness (NANDA)" OR MH "Social Alienation" OR MH "Support, Psychosocial" OR MH "Psychosocial Aspects of Illness" OR MH "Psychosocial Deprivation" OR MH "Happiness" OR MH "Hope" OR MH "Hopelessness" OR MH "Love" OR MH "Pleasure" OR MH "Personal Satisfaction" OR MH "Quality of Life" OR MH "Comfort" OR MH "Interpersonal Relations" OR MH "Friendship" OR MH "Self Concept" OR MH "Confidence" OR MH "Self-Efficacy" OR MH "Leisure Activities" OR MH "Recreation"

12. TI(particip* OR companion* OR leisure OR psychosocial OR (social W2 activit*) OR (social W2 behavior*) OR (social W2 connect*) OR (social W2 engagement*) OR (social W2 involvement*) OR (social W2 inclusion*) OR (social W2 contact*) OR interact* OR "quality of life" OR (life N2 satisfaction*) OR wellbeing OR relax* OR happiness*OR (positive W2 emotion*) OR (social W2 identit*) OR "self efficacy" OR salutogen* OR "health promotion" OR (health W2 prevention*) OR (illness W2 prevention) OR meaning OR distress* OR mood* OR loneliness OR boredom OR isolation* OR depress* OR anxiety) 
OR AB ((psychosocial W2 intervent*) OR companion* OR (social W2 activit*) OR (social W2 behavior*) OR (social W2 connect*) OR (social W2 engagement*) OR (social W2 involvement*) OR (social W2 inclusion*) OR particip* OR (social W2 contact*) OR (social W2 interaction*) OR particip* OR loneliness OR boredom OR isolation* OR "quality of life" OR (life N2 satisfaction*) OR wellbeing OR relax* OR happiness*OR (positive W2 emotion*) OR (social W2 identit*) OR "self efficacy" OR salutogen* OR distress*)

13. 11 OR 12

Systematic reviews, meta-analysis, meta-synthesis

14. (MH "Systematic Review" OR MH "Meta Analysis" OR MH "Meta Synthesis") OR TX ((systematic* N3 review*) OR (metaanaly* OR (meta W2 analy*)) OR TX ((overview* N2 (review* OR systematic OR synthes* OR summary OR umbrella OR (integrative W3 review) OR (information W2 synthes*) OR (data W2 synthes*) OR (data N2 extract*)) OR TX ((meta W2 synthes*) OR metasyntheses* OR (meta W2 ethnograph*) OR metaethnograph* OR (thematic W2 synthes*) OR "meta-study" OR "meta-summary" OR "critical interpretive syntheses" OR "qualitative cross-case analysis" OR "grounded theory synthesis" OR "meta-interpretation" OR "scoping review" OR "realist review" OR "mapping review" OR "systematic mapping"))

\begin{tabular}{clc}
\hline Limits: & & \\
\hline 15. & Limiters - Language: Danish, English, Norwegian, Swedish & \\
\hline 16. & TI ("young adults" OR "young people" OR child* OR adolescen* OR youth OR school* OR infant*) & \\
\hline Combined sets & 2688 \\
\hline 3 AND 7 & 137 \\
\hline 17 AND 14 & $\mathbf{1 3 2}$ \\
\hline 18 AND 15 & 6428 \\
\hline 3 AND 6 AND 10 AND 13 & 445 \\
\hline 20 AND 14 & $\mathbf{4 3 9}$
\end{tabular}

The search result, usually found at the end of the documentation, forms the list of abstracts.

$\mathrm{AB}=$ Abstract

$\mathrm{AU}=$ Author

$\mathrm{DE}=$ Term from the thesaurus

$\mathrm{MM}=$ Major Concept

$\mathrm{TI}=$ Title

TX = All Text. Performs a keyword search of all the isEpeidatabase's searchable fields

$\mathrm{ZC}=$ Methodology Index

$*$ Truncation

" " = Citation Marks; searches for an exact phrase

\section{IBSS via Proquest 18 April 2017}

Search terms

Population: Age

1. SU.EXACT("Aging") OR SU.EXACT("Older people") OR SU.EXACT("Oldest old people") OR

SU.EXACT("Frailty") OR SU.EXACT("Seniority") OR SU.EXACT("Senior citizen centers") OR

Items found

SU.EXACT("Retirees") OR SU.EXACT("Retirement homes") OR SU.EXACT("Retirement communities") OR

SU.EXACT("Elder care") OR SU.EXACT("Retirement") OR

SU.EXACT("Pensions")

2. ti(old* OR elder* OR aged* OR aging* OR ageing* OR (old PRE/1 patient*) OR (old PRE/1 age*) OR (old* 36825 PRE/1 people*) OR (older PRE/1 patient*) OR adult* OR senior* OR retire*) OR if(old* OR elder* OR aged* OR aging* OR ageing* OR (old PRE/1 patient*) OR (old PRE/1 age*) OR (old* PRE/1 people*) OR (older PRE/1 patient*) OR adult* OR senior* OR retire*)

3. 1 OR 2

\section{Intervention: Social robots}

4. SU.EXACT("Adaptive technology") OR ti((companion N/3 robot*) OR (social* N/3 robot*) OR (sociable N/3 robot*) OR (domestic PRE/2 robot*) OR (healthcare N/3 robot*) OR Paro OR (video PRE/2 game*) OR (assistive PRE/2 technolog*)) OR if((companion N/3 robot*) OR (social* N/3 robot*) OR (sociable N/3 robot*) OR (domestic PRE/2 robot*) OR (healthcare N/3 robot*) OR Paro OR (video PRE/2 game*) OR (virtual PRE/2 realit*) OR (virtual PRE/2 environment*) OR "science and technology studies" OR (assistive PRE/2 technolog*)) OR ab((companion N/3 robot*) OR (social*N/3 robot*) OR (sociable N/3 robot*) OR (domestic PRE/2 robot*) OR (healthcare N/3 robot*) OR Paro OR (video PRE/2 game*) OR (virtual PRE/2 realit*) OR (virtual PRE/2 environment*) OR "science and technology studies" OR (assistive PRE/2 technolog*))

\section{Intervention: ICT/Social media}

5. SU.EXACT("Text messaging") OR SU.EXACT("Chat rooms") OR SU.EXACT("Semantic web") OR SU.EXACT("Internet") OR SU.EXACT("Web studies") OR SU.EXACT("Information technology") OR SU.EXACT("Instant messaging") OR SU.EXACT("Wikis") OR SU.EXACT("Videophones") OR SU.EXACT("Internet telephony") OR SU.EXACT("Virtual communities") OR SU.EXACT("Cellular telephones") OR SU.EXACT("Computer \& video games") OR SU.EXACT("World Wide Web") OR SU.EXACT("Weblogs") OR SU.EXACT("Web sites") OR SU.EXACT("Telephones") OR SU.EXACT("Wireless communications") OR SU.EXACT("Electronic games")

6. $\quad$ ti((information N/2 technol*) OR ICT OR "science and technology studies" OR (virtual PRE/2 realit*) OR (virtual PRE/2 environment*) OR internet* OR computer* OR digital OR online OR (web W2 based*) OR (web W2 2.0) OR (social PRE/2 network*) OR twitter* OR facebook* OR "face book" OR blogging OR blogs OR (social PRE/2 media*) OR (electronic PRE/2 mail*) OR "e-mail" OR email* OR (text PRE/2 
messag*) OR sms OR mms* OR phone* OR telephone* OR android* OR iphone* OR smartphone* OR cellphone* OR mobile* OR skype OR apps* OR ipad* OR multimedia* OR (multi PRE/2 media*) OR technolog* OR video* OR digital*) OR if((information N/2 technol*) OR "science and technology studies" OR (virtual PRE/2 realit*) OR ((virtual PRE/2 environment*) OR internet* OR computer* OR digital OR online OR (web PRE/2 based*) OR (web PRE/2 2.0) OR (social PRE/2 network*) OR twitter* OR facebook* OR "face book" OR blogging OR blogs OR (social PRE/2 media*) OR (electronic PRE/2 mail*) OR "e-mail" OR email* OR (text PRE/2 messag*) OR sms OR mms* OR phone* OR telephone* OR android* OR iphone* OR smartphone* OR cellphone* OR mobile* OR skype OR apps* OR ipad* OR multimedia* OR (multi PRE/2 media*) OR technolog* OR video* OR ehealth)) OR ab("science and technology studies" OR (virtual PRE/2 realit*) OR (virtual PRE/2 environment*) OR internet* OR computer* OR digital OR online OR (web PRE/2 based*) OR (web PRE/2 2.0) OR (social PRE/2 network*) OR twitter* OR facebook* OR "face book" OR blogging OR blogs OR (social PRE/2 media*) OR (electronic PRE/2 mail*) OR "e-mail" OR email* OR (text PRE/2 messag*) OR sms OR mms* OR phone* OR telephone* OR android* OR iphone* OR smartphone* OR cellphone* OR (mobile PRE/2 phone*) OR apps* OR ipad* OR multimedia* OR (multi PRE/2 media*) OR (video PRE/2 game*) OR ehealth)

\section{5 OR 6}

\section{Mental health/Social isolation/Social interaction}

8. SU.EXACT("Mental depression") OR SU.EXACT("Anxieties") OR SU.EXACT("Participation") OR SU.EXACT("Social promotion") OR SU.EXACT("Alienation") OR SU.EXACT("Citizen participation") OR SU.EXACT("Social interaction") OR SU.EXACT("Social identity") OR SU.EXACT("Loneliness") OR SU.EXACT("Social isolation") OR SU.EXACT("Empowerment") OR SU.EXACT("Leisure")

9. ti(leisure OR companion* OR empower OR (psychosocial PRE/2 intervent*) OR (pro PRE/2 social) OR 111942 (social PRE/2 activit*) OR (social PRE/2 behavior*) OR (social PRE/2 connect*) OR (social PRE/2 contact*) OR (social PRE/2 interaction*) OR (social PRE/2 engagement*) OR (social PRE/2 involvement*) OR (social PRE/2 inclusion*) OR (social PRE/2 identit*) OR efficacy OR particip* OR loneliness OR boredom OR isolation* OR "quality of life" OR (life N/2 satisfaction*) OR wellbeing OR relax* OR happiness* OR (positive PRE/2 emotion*) OR meaning OR distress* OR mood* OR depress* OR anxiety OR salutogen* OR "health promotion" OR (health PRE/2 prevention*) OR (illness PRE/2 prevention)) OR if(leisure OR companion* OR empower OR (psychosocial PRE/2 intervent*) OR (pro PRE/2 social) OR (social PRE/2 activit*) OR (social PRE/2 behavior*) OR (social PRE/2 connectt*) OR (social PRE/2 contact*) OR (social PRE/2 interaction*) OR (social PRE/2 engagement*) OR (social PRE/2 involvement*) OR (social PRE/2 inclusion*) OR (social PRE/2 identit*) OR efficacy OR particip* OR loneliness OR boredom OR isolation* OR "quality of life" OR (life N/2 satisfaction*) OR wellbeing OR relax* OR happiness*OR (positive PRE/2 emotion*) OR meaning OR distress* OR mood* OR depress* OR anxiety OR salutogen* OR "health promotion" OR (health PRE/2 prevention*) OR (illness PRE/2 prevention))OR ab((psychosocial PRE/2 intervent*) OR companion* OR (social PRE/2 activit*) OR (social PRE/2 behavior*) OR (social PRE/2 connect*) OR (social PRE/2 contact*) OR (social PRE/2 interaction*) OR (social PRE/2 engagement*) OR (social PRE/2 involvement*) OR (social PRE/2 inclusion*) OR (social PRE/2 identit*) OR efficacy OR particip* OR loneliness OR boredom OR isolation* OR "quality of life" OR (life N/2 satisfaction*) OR wellbeing OR relax* OR happiness*OR (positive PRE/2 emotion*) OR distress* OR depress* OR anxiety OR salutogen* OR "health promotion" OR (health PRE/2 prevention*) OR (illness PRE/2 prevention))

Systematic reviews, meta-analysis, meta-synthesis

11. (SU.EXACT("Systematic reviews" OR "Meta-analysis") OR ti((systematic* N/3 review*) OR metaanaly* OR 3021 (meta PRE/2 analy*) OR (technology PRE/2 overview*) OR (technology PRE/2 appraisal*) OR (technology PRE/2 assessment*) OR (overview* N/2 (review* OR systematic OR synthes* OR summary OR umbrella)) OR (integrative PRE/2 review) OR (information PRE/2 synthes*) OR (data PRE/2 synthes*) OR (data N/2 extract*) OR (systematic PRE/2 synthes*) OR (meta PRE/2 synthes*) OR metasyntheses* OR (meta PRE/2 ethnograph*) OR metaethnograph* OR (thematic PRE/2 synthes*) OR "meta-study" OR "meta-summary" OR "critical interpretive syntheses" OR "qualitative cross-case analysis" OR "grounded theory synthesis" OR "meta-interpretation") OR if((systematic* N/3 review*) OR metaanaly* OR (meta PRE/2 analy*) OR (technology PRE/2 overview*) OR (technology PRE/2 appraisal*) OR (technology PRE/2 assessment*) OR (overview* N/2 (review* OR systematic OR synthes* OR summary OR umbrella)) OR (integrative PRE/2 review) OR (information PRE/2 synthes*) OR (data PRE/2 synthes*) OR (data N/2 extract*) OR (systematic PRE/2 synthes*) OR (meta PRE/2 synthes*) OR metasyntheses* OR (meta PRE/2 ethnograph*) OR metaethnograph* OR (thematic PRE/2 synthes*) OR "meta-study" OR "meta-summary" OR "critical interpretive syntheses" OR "qualitative cross-case analysis" OR "grounded theory synthesis" OR "metainterpretation") OR ab ((systematic* N/3 review*) OR metaanaly* OR (meta PRE/2 analy*) OR (technology PRE/2 overview*) OR (technology PRE/2 appraisal*) OR (technology PRE/2 assessment*) OR (overview* N/2 (review* OR systematic OR synthes* OR summary OR umbrella)) OR (integrative PRE/2 review) OR (information PRE/2 synthes*) OR (data PRE/2 synthes*) OR (data N/2 extract*) OR (systematic PRE/2 synthes*) OR (meta PRE/2 synthes*) OR metasyntheses* OR (meta PRE/2 ethnograph*) OR metaethnograph* OR (thematic PRE/2 synthes*) OR "meta-study" OR "meta-summary" OR "critical interpretive syntheses" OR "qualitative cross-case analysis" OR "grounded theory synthesis" OR "metainterpretation"))

\section{Combined sets}

The search result, usually found at the end of the documentation, forms the list of abstracts.

$\mathrm{AB}=$ Abstract

$\mathrm{AU}=$ Author 
$\mathrm{DE}=$ Term from the thesaurus

$\mathrm{MM}=$ Major Concept

$\mathrm{TI}=$ Title

TX = All Text. Performs a keyword search of all the sispidatabase's searchable fields

ZC $=$ Methodology Index

$*$ Truncation

$"$ " = Citation Marks; searches for an exact phrase

Psyclnfo via EBSCO 3 September 2019

\section{Search terms}

Population: Age

1. (ZG "aged (65 yrs \& older)") or (ZG "very old (85 yrs \& older)") OR DE "Aging" OR DE "Retirement" OR DE 344639

"Gerontology" OR DE "Retirement" OR DE "Ageism" OR DE "Aged (Attitudes Toward)" OR DE "Late Life

Depression") OR DE "Geropsychology"

2. $\quad$ TI(old* OR elder* OR aged* OR aging* OR ageing* OR adult* OR senior* OR retire*) OR KW(old*W2 age*) OR older OR (middle W2 age*) OR middleage* OR (old* W2 patient*) OR (old* W2 people*) OR (old* W2 person*) OR elder* OR aged OR aging OR ageing* OR senior* OR retire*)

3. 1 OR 2
1 OR 2

\section{Intervention: Social robots}

4. DE "Assistive Technology" OR TI((companion N3 robot*) OR (social* N3 robot*) OR (sociable N2 robot*) 1946 OR (domestic W2 robot*) OR (healthcare N3 robot*) OR Paro OR (video W2 game*) OR (assistive W2 technolog*) OR gerontechnology) OR AB((companion N3 robot*) OR (social* N3 robot*) OR (sociable N2 robot*) OR (domestic W2 robot*) OR (healthcare N3 robot*) OR Paro OR (video W2 game*) OR "science and technology studies" OR (assistive W2 technolog*) OR gerontechnology) OR KW((companion N3 robot*) OR (social* N3 robot*) OR (sociable N2 robot*) OR (domestic W2 robot*) OR (healthcare N3 robot*) OR Paro OR (video W2 game*) OR (assistive W2 technolog*) OR gerontechnology)

\section{Intervention: ICT/Social media}

5. DE "Information Technology" OR DE "Communications Media" OR DE "Audiovisual Communications Media" OR DE "Multimedia" OR DE "Telecommunications Media" OR DE "Electronic Communication" OR DE "Blog" OR DE "Computer Mediated Communication" OR DE "Electronic Learning" OR DE "Social Media" OR DE "Online Social Networks" OR DE "Online Community") OR DE "Text Messaging" OR DE "Internet" OR DE "Mobile Devices" OR DE "Cellular Phones" OR DE "Electronic Communication" OR DE "Video Display Units" OR DE "Computer Peripheral Devices" OR DE "Computers" OR DE "Virtual Reality" OR DE "Computer Simulation" OR DE "Computer Applications" OR DE "Human Machine Systems" OR DE "Computer Games" OR DE "Role Playing Games" OR DE "Telemedicine"

6. TI ((information N3 technol*) OR ICT OR (virtual W2 realit*) OR (virtual W2 environment*) OR internet* OR computer* OR digital* OR online* OR (web W2 based) OR (web W2 2.0) OR (social W2 network*) OR "social network site" OR twitter* OR facebook* OR (face W1 book*) OR blogging OR blogs OR (social W2 media*) OR (electronic W2 mail*) OR "e-mail*" OR email* OR (text W2 messag*) OR sms OR mms* OR phone* OR telephone* OR android* OR iphone* OR smartphone* OR cellphone* OR mobile* OR skype OR apps* OR ipad* OR multimedia* OR (multi W2 media*) OR technolog* OR video* OR digital OR ehealth) OR KW((information W2 technol*) OR "science and technology studies" OR (virtual W2 realit*) OR (virtual W2 environment*) OR internet* OR computer* OR digital OR online OR (web W2 based) OR (web W2 2.0) OR (social W2 networking*) OR "social network site" OR twitter* OR facebook* OR (face W1 book*) OR blogging OR blogs OR (social W2 media*) OR (electronic W2 mail*) OR "email*" OR email* OR (text W2 messag*) OR phone* OR telephone* OR android* OR iphone* OR smartphone* OR cellphone* OR mobile* OR skype OR apps* OR ipad* OR multimedia* OR (multi W2 media*) OR (video W2 game*) OR digital OR ehealth)

\section{Mental health/Social isolation/Social interaction}

8. $\quad$ DE "Social Deprivation" OR DE "Stimulus Deprivation" OR DE "Social Isolation" OR DE "Anxiety" OR DE 404647 "Social Anxiety" OR DE "Emotional States" OR DE "Alienation" OR DE "Apathy" OR DE "Aversion" OR DE "Depression (Emotion)" OR DE "Dissatisfaction" OR DE "Distress" OR DE "Emotional Trauma" OR DE "Enthusiasm" OR DE "Frustration" OR DE "Happiness" OR DE "Helplessness" OR DE "Hope" OR DE "Hopelessness" OR DE "Loneliness" OR DE "Pleasure" OR DE "Sadness" OR DE "Emotional Assessment" OR DE "Emotional Disturbances" OR DE "Positive Emotions" OR DE "Recreation" OR DE "Relaxation" OR DE "Life Satisfaction" OR DE "Satisfaction" OR DE "Quality of Life" OR DE "Well Being" OR DE "Positive Psychology" OR DE "Optimism" OR DE "Positive Emotions" OR DE "Social Interaction" OR DE "Online Social Networks" OR DE "Self-Efficacy" OR DE "Self-Concept"

9. $\quad \mathrm{TI}($ particip* OR companion* OR leisure OR psychosocial OR (social W2 activit*) OR (social W2 behavior*) OR (social W2 activit*) OR (social W2 connect*) OR (social W2 engagement*) OR (social W2 involvement*) OR (social W2 inclusion*) OR (social W2 contact*) OR interact* OR "quality of life" OR (life N2 satisfaction*) OR wellbeing OR relax* OR happiness*OR (positive W2 emotion*) OR (social W2 identit*) OR "self efficacy" OR salutogen* OR "health promotion" OR (health W2 prevention*) OR (illness W2 prevention) OR meaning OR distress* OR mood* OR loneliness OR boredom OR isolation* OR depress* OR anxiety) OR KW(particip* OR companion* OR leisure OR psychosocial OR (social W2 activit*) OR (social W2 behavior*) OR (social W2 activit*) OR (social W2 connect*) OR (social W2 engagement*) OR (social W2 involvement*) OR (social W2 inclusion*) OR (social W2 contact*) OR interact* OR "quality of life" OR (life N2 satisfaction*) OR wellbeing OR relax* OR happiness*OR (positive W2 emotion*) OR (social W2 identit*) OR "self efficacy" OR salutogen* OR "health promotion" OR (health W2 prevention*) OR (illness W2 prevention) OR meaning OR distress* OR mood* OR loneliness OR boredom OR isolation* OR depress* OR anxiety) 
Study types: Systematic reviews, meta-analysis, meta-synthesis

11. DE "Meta Analysis" OR ZC "systematic review" OR ZC "meta analysis" OR TX ((systematic* N3 review*) 71752

OR (metaanaly* OR (meta W2 analy*) OR (technology W2 overview*) OR (technology W2 appraisal*) OR (technology W2 assessment*)) OR TX ((overview* N2 (review* OR systematic OR synthes* OR summary OR umbrella)) OR TX((integrative W3 review) OR (information W2 synthes*) OR (data W2 synthes*) OR (data N2 extract*)) OR JN ("Cochrane Database of Systematic Reviews") OR TX ((systematic W2 synthes*) OR (meta W2 synthes*) OR metasyntheses* OR (meta W2 ethnograph* OR metaethnograph* OR (thematic W2 synthes*) OR "meta-study" OR "meta-summary" OR "critical interpretive syntheses" OR "qualitative cross-case analysis" OR "grounded theory synthesis" OR "meta-interpretation" OR "scoping review" OR "realist review" OR "mapping review" OR "systematic mapping"))

Limits:

12. Limiters - Language: Danish, English, Norwegian, Swedish

\begin{tabular}{cc}
\hline Combined sets & 2027 \\
\hline 3 AND 4 & $\mathbf{9 3}$ \\
\hline 13 AND 11 & $\mathbf{9 1}$ \\
\hline 14 AND 12 & $\mathbf{7 3 8 6}$ \\
\hline 3 AND 7 AND 10 & $\mathbf{1 5 8}$ \\
\hline 16 AND 11 & $\mathbf{1 5 4}$
\end{tabular}

The search result, usually found at the end of the documentation, forms the list of abstracts.

$\mathrm{AB}=$ Abstract

$\mathrm{AU}=$ Author

$\mathrm{DE}=$ Term from the thesaurus

$M M=$ Major Concept

$\mathrm{TI}=$ Title

TX = All Text. Performs a keyword search of all the isepidatabase's searchable fields

$\mathrm{ZC}=$ Methodology Index

$*$ Truncation

$"$ " = Citation Marks; searches for an exact phrase

PubMed via NLM 2 September 2019

Search terms

Population: Age

1. (("Aged"[Mesh] OR "Aged, 80 and over"[Mesh] OR "Frail Elderly"[Mesh] OR "Homes for the Aged" [MeSH] 3362812

OR "Housing for the Elderly"[Mesh] OR "Aging"[Mesh] OR "Retirement" [Mesh] OR old*[Title] OR

retired[Title] OR retirement*[Title] OR elder*[Title] OR aged*[Title] OR aging*[Title]) OR

((aging*[Title/Abstract] OR ageing*[Title/Abstract] OR elder*[Title/Abstract] OR aged

people*[Title/Abstract] OR aged person*[Title/Abstract] OR aged women*[Title/Abstract] OR aged

men*[Title/Abstract] OR old patient*[Title/Abstract] OR old age*[Title/Abstract] OR old

people*[Title/Abstract] OR old people*[Title/Abstract] OR old person*[Title/Abstract] OR old

women*[Title/Abstract] OR old men*[Title/Abstract] OR older age*[Title/Abstract] OR older

people*[Title/Abstract] OR older patient*[Title/Abstract] OR older adult*[Title/Abstract] OR

senior*[Title/Abstract] OR retired[Title/Abstract] OR retirement*[Title/Abstract] OR old*[Other Term] OR

aging*[Other Term] OR elder*[Other Term] OR aged people*[Other Term] OR aged person*[Other Term]

OR aged women*[Other Term] OR aged men*[Other Term] OR senior*[Other Term] OR retirement*[Other Term] ) NOT medline $[\mathrm{SB}])$ )

Population: Mental health

2. (("Mental Health"[Mesh] OR "Mental Disorders"[Mesh] OR "Depression"[Mesh] OR "Social

Isolation"[Mesh] OR "Loneliness"[Mesh] OR mental[Title] OR depress*[Title] OR anxiet*[Title] OR isolation[Title] OR loneliness[Title]) OR ((mental health*[Title/Abstract] OR mental

disorder*[Title/Abstract] OR depression*[Title/Abstract] OR depressive*[Title/Abstract] OR anxiety[Title/Abstract] OR eating disorder*[Title/Abstract] OR anorexi*[Title/Abstract] OR mood disorder*[Title/Abstract] OR maladaptive stress*[Title/Abstract] OR emotional exhaustion*[Title/Abstract] OR mental exhaustion*[Title/Abstract] OR dysthym*[Title/Abstract] OR mental problem*[Title/Abstract] OR mental wellness[Title/Abstract] OR mental well-being[Title/Abstract] OR mental [Other Term] OR depress*[Other Term] OR anxiet*[Other Term] OR mood [Other Term]) NOT Medline[SB]))

\section{Intervention: Social robots}

3. companion $\operatorname{robot}^{*}[$ Title/Abstract] OR social robot*[Title/Abstract] OR robotic companion*[Title/Abstract] OR social assistive robot*[Title/Abstract] OR socially assistive robot*[Title/Abstract] OR socially interactive robot*[Title/Abstract] OR socially intelligent robot*[Title/Abstract] OR sociable robot*[Title/Abstract] OR socializing robot*[Title/Abstract] OR domestic robot*[Title/Abstract] OR healthcare robot*[Title/Abstract] OR Paro[Title/Abstract] OR CareTV[Title/Abstract] OR science and technology studies[Title/Abstract] OR assistive technolog*[Title/Abstract] OR relational agent*[Title/Abstract] OR welfare technol*[Title/Abstract] OR gerontechnology [Title/Abstract] OR ((companion[Title/Abstract] OR socia*[Title/Abstract] OR interactive*[Title/Abstract]) AND technol*[Title/Abstract]) OR companion robot*[Other Term] OR social robot*[Other Term] OR socially assistive robot*[Other Term] OR robotic companion*[Other Term] OR social assistive robot*[Other Term] OR socially interactive robot*[Other Term] OR socially intelligent robot*[Other Term] OR sociable robot*[Other Term] OR socializing robot*[Other Term] OR domestic robot*[Other Term] OR healthcare robot*[Other Term] OR Paro[Other Term] OR CareTV[Other Term] OR science and technology studies [Other Term] OR assistive technolog*[Other Term] OR relational agent*[Other Term] OR gerontechnology[Other Term] 
4. "Information Science"[Mesh] OR "Computer Literacy"[Mesh] OR "Internet"[Mesh] OR "Blogging"[Mesh]

OR "Social media"[Mesh] OR "Computers"[Mesh] OR "Electronic Mail"[Mesh] OR "Text Messaging"[Mesh] OR "Audiovisual Aids"[Mesh] OR "Telephone"[Mesh] OR "Answering Services" [Mesh] OR "Cell Phones" [Mesh] OR "Smartphone"[ [Mesh] OR "Television" [Mesh] OR "Wireless Technology" [Mesh] OR "Video Games"[Mesh]

5. technolog*[Title] OR digital*[Title] OR online[Title] OR mobile[Title] OR game[Title] OR games[Title] OR information technol*[Title/Abstract] OR care technolog* OR virtual realit*[Title/Abstract] OR virtual environ*[Title/Abstract] OR internet*[Title/Abstract] OR computer*[Title/Abstract] OR web based*[Title/Abstract] OR web 2.0[Title/Abstract] OR social networking*[Title/Abstract] OR social network site*[Title/Abstract] OR chat[Title/Abstract] OR chats[Title/Abstract] OR chatting [Title/Abstract] OR twitter*[Title/Abstract] OR facebook*[Title/Abstract] OR face book*[Title/Abstract] OR social media*[Title/Abstract] OR electronic mail*[Title/Abstract] OR email*[Title/Abstract] OR email*[Title/Abstract] OR text messag*[Title/Abstract] OR phone*[Title/Abstract] OR telephone*[Title/Abstract] OR android*[Title/Abstract] OR iphone*[Title/Abstract] OR smartphone*[Title/Abstract] OR cellphone*[Title/Abstract] OR mobile phone*[Title/Abstract] OR skype[Title/Abstract] OR apps[Title/Abstract] OR ipad*[Title/Abstract] OR multimedia*[Title/Abstract] OR multi media*[Title/Abstract] OR video game*[Title/Abstract] OR gaming[Title/Abstract] OR ehealth[Title/Abstract] OR care technolog*[Other Term] OR information technol*[Other Term] OR ict[Other Term] OR care technolog*[Other Term] OR virtual realit*[Other Term] OR virtual realit*[Other Term] OR virtual environment* [Other Term] OR digital [Other Term] OR internet* [Other Term] OR computer*[Other Term] OR online[Other Term] OR web based*[Other Term] OR web 2.0[Other Term] OR social networking*[Other Term] OR social network site*[Title/Abstract] OR chat[Other Term] OR chats[Other Term] OR twitter*[Other Term] OR facebook*[Other Term] OR face book*[Other Term] OR social media*[Other Term] OR electronic mail*[Other Term] OR e-mail*[Other Term] OR email*[Other Term] OR text messag*[Other Term] OR sms[Other Term] OR mms*[Other Term] OR phone*[Other Term] OR telephone*[Other Term] OR android*[Other Term] OR iphone*[Other Term] OR smartphone*[Other Term] OR cellphone*[Other Term] OR mobile*[Other Term] OR skype[Other Term] OR apps[Other Term] OR ipad*[Other Term] OR multimedia*[Other Term] OR multi media*[Other Term] OR video*[Other Term] OR game[Other Term] OR games [Other Term ]OR gaming[Other Term] OR ehealth [Other Term]

$6 . \quad 4$ OR 5

\section{Social isolation/Social interaction}

7. "Social Participation"[Mesh] OR "Social Isolation"[Mesh] OR "Loneliness"[Mesh] OR "Social Alienation"[Mesh] OR "Social Behavior"[Mesh] OR "Social Marginalization"[Mesh] OR "Psychosocial Support Systems" [Mesh] OR "Personal Satisfaction" [Mesh] OR "Social Determinants of Health"[Mesh] OR "Social Identification" [Mesh] OR "Self Concept"[Mesh] OR "Self Efficacy"[Mesh] OR "Sense of Coherence" [Mesh] OR "Role"[Mesh:NoExp] OR "Community Participation"[Mesh]

8. participat*[Title] OR meaning[Title] OR mood[Title] OR moody[Title] OR leisure[Title/Abstract] OR companion*[Title/Abstract] OR pro-social[Title/Abstract] OR social activit*[Title/Abstract] OR community participation*[Title/Abstract] OR community involv*[Title/Abstract] OR social connect*[Title/Abstract] OR sense of belonging [Title/Abstract] OR social involve* [Title/Abstract] OR social inclusion*[Title/Abstract] OR social contact*[Title/Abstract] OR interact*[Title/Abstract] OR (social[Title/Abstract] AND participat*[Title/Abstract]) OR quality of life[Title/Abstract] OR life satisfaction[Title/Abstract] OR wellbeing[Title/Abstract] OR relax*[Title/Abstract] OR positive emotion*[Title/Abstract] OR social identit*[Title/Abstract] OR self efficacy[Title/Abstract] OR salutogen*[Title/Abstract] OR health promotion[Title/Abstract] OR health prevention*[Title/Abstract] OR illness prevention[Title/Abstract] OR loneliness[Title/Abstract] OR isolation[Title/Abstract] OR distress*[Title/Abstract] OR boredom[Title/Abstract] OR psychosocial intervent*[Other Term] OR companion*[Other Term] OR prosocial [Other Term] OR social activit*[Other Term] OR social behavior*[Other Term] OR community participation*[Other Term] OR community involv*[Other Term] OR social connect*[Other Term] OR social engagement*[Other Term] OR social involve* [Other Term] OR social inclusion*[Other Term] OR social contact*[Other Term] OR social interaction*[Other Term] OR interact*[Other Term] OR particip*[Other Term] OR quality of life[Other Term] OR life satisfaction[Other Term] OR meaning[Other Term] OR wellbeing [Other Term] OR relax*[Other Term] OR positive emotion*[Other Term] OR meaning[Other Term] OR social identit*[Other Term] OR self efficacy[Other Term] OR mood[Other Term] OR moody[Other Term] OR loneliness[Other Term] OR isolation[Other Term] OR distress*[Other Term] OR boredom[Other Term]

9. $\quad 7$ OR 8

\section{Study types: Systematic reviews, meta-analysis, meta-synthesis}

10. Systematic[SB] OR (systematic synthesis[Title/Abstract] OR meta synthesis[Title/Abstract] OR metasynthesis[Title/Abstract] OR qualitative synthesis[Title/Abstract] OR meta ethnography [Title/Abstract]
OR metaethnography[Title/Abstract] OR meta study [Title/Abstract] OR metastudy [Title/Abstract] OR meta summary[Title/Abstract] OR metasummary[Title/Abstract] OR thematic synthesis[Title/Abstract] OR critical interpretive synthesis[Title/Abstract] OR grounded theory synthesis[Title/Abstract] OR meta interpretation [Title/Abstract] OR cross case analysis[Title/Abstract] OR scoping review[Title/Abstract] OR realist review*[Title/Abstract] OR mapping review*[Title/Abstract] OR systematic mapping [Title/Abstract]) OR (systematic synthesis[Other Term] OR meta synthesis[Other Term] OR metasynthesis[Other Term] OR qualitative synthesis[Other Term] OR meta ethnography[Other Term] OR metaethnography[Other Term] OR meta study[Other Term] OR metastudy[Other Term] OR meta summary[Other Term] OR metasummary[Other Term] OR thematic synthesis[Other Term] OR critical interpretive synthesis[Other Term] OR grounded theory synthesis[Other Term] OR meta interpretation [Other Term] OR cross case analysis [Other Term] OR scoping review*[Other Term] OR realist review*[Other Term] OR mapping review*[Other Term] OR systematic mapping[Other Term]) 
Combined sets

\begin{tabular}{clr}
\hline 11. & 1 AND 3 & 2727 \\
\hline 12. & 11 AND 10 & $\mathbf{1 0 7}$ \\
\hline 13. & 1 AND 2 AND 6 AND 9 & 9013 \\
\hline & 13 AND 10 & $\mathbf{9 9}$ \\
\hline
\end{tabular}

The search result, usually found at the end of the documentation, forms the list of abstracts.

$[\mathrm{MeSH}]=$ Term from the Medline controlled vocabulary, including terms found below this term in the MeSH hierarchy

$[\mathrm{MeSH}: \mathrm{NoExp}]=$ Does not include terms found below this term in the MeSH hierarchy

$[\mathrm{MAJR}]=$ MeSH Major Topic

$[$ Title/Abstract $]=$ Title or abstract

$[\mathrm{TI}]=$ Title

$[\mathrm{AU}]=$ Author

$[\mathrm{OT}]=$ Other term

$[\mathrm{TW}]=$ Text Word

Systematic $[\mathrm{SB}]=$ Filter for retrieving systematic reviews

$*=$ Truncation

\begin{tabular}{|c|c|c|}
\hline \multicolumn{3}{|c|}{ Scopus via Elsevier 2 September 2019} \\
\hline \multicolumn{2}{|c|}{ Search terms } & Items found \\
\hline \multicolumn{3}{|c|}{ Population: Age } \\
\hline 1. & $\begin{array}{l}\text { INDEXTERMS ("Aged" OR "Homes for the Aged" OR "Housing for the Elderly") OR TITLE-ABS-KEY ((old* } \\
\text { PRE/ } 1 \text { people) OR (old* PRE/1 patient*) OR (old* PRE/1 age*) OR aging* OR ageing* OR elder* OR (aged } \\
\text { PRE/1 people*) OR (aged PRE/1 person*) OR (aged PRE/1 women*) OR (aged PRE/1 men) OR senior* OR } \\
\text { retired OR retirement) }\end{array}$ & 5601054 \\
\hline \multicolumn{3}{|c|}{ Intervention: Social robot } \\
\hline 2. & $\begin{array}{l}\text { TITLE-ABS-KEY ((assistive PRE/2 technolog*) OR (companion PRE/2 robot*) OR (social PRE/2 robot*) OR } \\
\text { (robotic PRE/2 companion*) OR (socially W/2 robot*) OR (sociable PRE/2 robot*) OR (socializing PRE/2 } \\
\text { robot*) OR (domestic PRE/2 robot*) OR (healthcare PRE/2 robot*) OR Paro OR "science technology } \\
\text { studies") }\end{array}$ & 16537 \\
\hline \multicolumn{3}{|c|}{ Intervention: ICT /social media } \\
\hline 3. & $\begin{array}{l}\text { INDEXTERMS ("Information Science" OR "Computer Literacy" OR "Internet" OR "Blogging" OR "Social } \\
\text { media" OR "Computers" OR "Electronic Mail" OR "Text Messaging" OR "Audiovisual Aids" OR } \\
\text { "Telephone" OR "Answering services" OR "Cell Phones" OR "Smartphone" OR "Text messaging" OR } \\
\text { "Television" OR "Wireless Technology" OR "Video Games") OR TITLE-ABS-KEY (internet* OR computer* } \\
\text { OR "social network site" OR chat OR chats OR twitter OR facebook* OR "face book" OR blogging OR } \\
\text { blogs OR "social media" OR "electronic mail" OR email* OR "e-mail" OR phone* OR telephone* OR } \\
\text { android* OR iphone* OR smartphone* OR cellphone* OR mobile* OR skype OR apps OR ipad* OR } \\
\text { multimedia* OR "multi media") OR TITLE-ABS-KEY ((information PRE/2 technol*) OR (virtual PRE/2 realit*) } \\
\text { OR (virtual PRE/2 environ*) OR (web PRE/1 based*) OR (web PRE/1 2.0) OR (social PRE/1 network*) OR } \\
\text { (text PRE/1 messag*) OR (video PRE/2 game*)) OR TITLE (technolog* OR digital OR online OR ehealth) }\end{array}$ & 6878192 \\
\hline \multicolumn{3}{|c|}{ Mental health/Social isolation/Social interaction } \\
\hline 4. & $\begin{array}{l}\text { INDEXTERMS ("Mental Health" OR "Mental Disorders" OR "Depression" OR "Social Participation" OR } \\
\text { "Social Isolation" OR "Loneliness" OR "Social Alienation" OR "Social Behavior" OR "Social } \\
\text { Marginalization" OR "Psychosocial Support Systems" OR "Personal Satisfaction" OR "Social Determinants } \\
\text { of Health" OR "Social Identification" OR "Self Concept" OR "Self Efficacy" OR "Sense of Coherence" OR } \\
\text { "Role" OR "Community Participation") OR TITLE(companion* OR leisure OR interact* OR particip* OR } \\
\text { loneliness OR boredom OR isolation* OR "quality of life" OR distress* OR wellbeing OR relax* OR } \\
\text { salutogen* OR efficacy OR depress* OR anxiety OR (social PRE/2 activit*) OR (social PRE/2 behavior*) } \\
\text { OR (social PRE/2 connect*) OR (social PRE/2 contact*) OR (social PRE/2 interaction*) OR (life PRE/2 } \\
\text { satisfaction*) OR (positive PRE/2 emotion*) OR (self PRE/2 concept*) OR (social PRE/2 role*) OR } \\
\text { (community PRE/2 participation*)OR (community PRE/2 involv*) OR "sense of belonging" OR (social } \\
\text { PRE/2 involve*) OR (social PRE/2 inclusion*)) OR ABS((social PRE/2 participation*) OR isolation OR } \\
\text { loneliness OR salutogen* OR efficacy OR depress* OR anxiety) }\end{array}$ & 3929395 \\
\hline
\end{tabular}

Study types: Systematic reviews, meta-analysis, meta-synthesis

5. INDEXTERMS("Meta-Analysis") OR TITLE-ABS-KEY ((systematic W/2 review*) OR (meta PRE/1 analy*) OR 401896 (systematic PRE/2 overview*) OR (integrative PRE/1 review) OR (meta PRE/1 synthes*) OR (meta PRE/1 ethnograph*) OR (thematic PRE/1 synthes*) OR metaanaly* OR metasyntheses* OR metaethnograph* OR "meta-study" OR "meta-summary" OR "critical interpretive syntheses" OR "qualitative cross-case analysis" OR "grounded theory synthesis" OR "meta-interpretation")

\begin{tabular}{|c|c|c|}
\hline \multicolumn{3}{|l|}{ Limits } \\
\hline 6. & TITLE(child* OR infant* OR adolescen* OR school* OR youth* OR young*) & \\
\hline \multicolumn{3}{|c|}{ Combined sets } \\
\hline 7. & 1 AND 2 AND 5 & 7 \\
\hline 8. & 1 AND 3 AND 4 AND 5 & 766 \\
\hline 9. & 8 NOT 6 & 755 \\
\hline
\end{tabular}

The search result, usually found at the end of the documentation, forms the list of abstracts.

TITLE-ABS-KEY = Title or abstract or keywords

ALL $=$ All fields

$\mathbf{P R E} / \mathbf{n}=$ "precedes by". The first term in the search must precede the second by a specified number of terms (n).

$\mathbf{W} / \mathbf{n}=$ "within". The terms in the search must be within a specified number of terms $(\mathrm{n})$ in any order.

$*=$ Truncation

" " = Citation Marks; searches for an exact phrase 
LIMIT-TO (SRCTYPE, "j" = Limit to source type journal

LIMIT-TO (DOCTYPE, "ar" = Limit to document type article

LIMIT-TO (DOCTYPE, "re" = Limit to document type review

\begin{tabular}{|c|c|c|}
\hline \multicolumn{3}{|c|}{ SocIndex via EBSCO 2 September 2019} \\
\hline Search & & Items found \\
\hline \multicolumn{3}{|c|}{ Population: Age } \\
\hline 1. & $\begin{array}{l}\text { DE "AGING" OR DE "ACTIVE aging" OR DE "OLDER people" OR DE "OLDER women" OR DE "OLDER } \\
\text { men" OR DE "OLD age" OR DE "AT-risk older people" OR DE "RETIREMENT" OR DE "EARLY retirement" } \\
\text { OR DE "PHASED retirement" OR DE "RETIREES" OR DE "SENIOR housing" OR DE "RETIREMENT } \\
\text { communities" OR DE "Geropsychology" }\end{array}$ & 27047 \\
\hline 2. & $\begin{array}{l}\text { TI(old* OR elder* OR older OR aged* OR aging* OR ageing* OR adult* OR senior* OR retire*) OR } \\
\text { KW(old* W2 age*) OR (old* W2 patient*) OR (old* W2 people*) OR (old* W2 person*) OR elder* OR } \\
\text { older OR aged OR aging OR ageing* OR senior* OR retire*) } \\
\text { OR AB(old* W2 age*) OR (old* W2 patient*) OR (old* W2 people*) OR elder* OR aged OR aging OR } \\
\text { ageing* OR senior* OR retirement*) }\end{array}$ & 171379 \\
\hline 3. & 1 OR 2 & 171606 \\
\hline
\end{tabular}

\section{Intervention: Social robots}

4. DE "ASSISTIVE computer technology" OR TI((companion N3 robot*) OR (social* N3 robot*) OR (sociable

N2 robot*) OR (domestic W2 robot*) OR (healthcare N3 robot*) OR Paro OR (assistive W2 technolog*) gerontechnology) OR KW((companion N3 robot*) OR (social* N3 robot*) OR (sociable N2 robot*) OR (domestic W2 robot*) OR (healthcare N3 robot*) OR Paro OR (assistive W2 technolog*) OR gerontechnology) OR AB((companion N3 robot*) OR (social* N3 robot*) OR (sociable N2 robot*) OR (domestic W2 robot*) OR (healthcare N3 robot*) OR Paro OR "science and technology studies" OR (assistive W2 technolog*) OR gerontechnology)

\section{Intervention: ICT/Social media}

5. DE "INFORMATION technology" OR DE "COMPUTER networks" OR DE "COMPUTERS -- Social aspects" OR (DE "COMPUTERS \& older people" OR DE "DIGITAL communications" OR DE

"TELECOMMUNICATION -- Social aspects" OR DE "TELEPHONE" OR DE "VIRTUAL communications" OR DE "VIRTUAL communities" OR DE "WEBCASTING" OR DE "CELL phones -- Social aspects" OR DE "SOCIAL networking mobile apps" OR DE "WEB 2.0" OR DE "WEBSITES" OR DE "SOCIAL media" OR DE "ONLINE comments" OR DE "ELECTRONIC discussion groups" OR DE "ONLINE chat" OR DE "BLOGS" OR DE "MICROBLOGS" OR DE "BLOGGERS" OR DE "VIDEO games" OR DE "VIDEO games for women"

6. TI ((information N3 technol*) OR ICT OR (virtual W2 realit*) OR (virtual W2 environment*) OR internet* OR computer* OR digital* OR online* OR (web W2 based*) OR (web W2 2.0) OR (social W2 network*) OR twitter* OR facebook* OR (face W1 book*) OR blogging OR blogs OR (social W2 media*) OR (electronic W2 mail*) OR "e mail" OR email* OR (text W2 messag*) OR sms OR mms* OR phone* OR telephone* OR android* OR iphone* OR smartphone* OR cellphone* OR mobile* OR skype OR apps* OR ipad* OR multimedia* OR (multi W2 media*) OR technolog* OR video* OR ehealth) OR KW((information W2 technol*) OR (virtual W2 realit*) OR (virtual W2 environment*) OR internet* OR computer* OR digital OR online OR (web W2 based*) OR (web W2 2.0) OR (social W2 network*) OR "social network site" OR twitter* OR facebook* OR (face W1 book*) OR blogging OR blogs OR (social W2 media*) OR (electronic W2 mail*) OR "e-mail" OR email* OR (text W2 messag*) OR phone* OR telephone* OR android* OR iphone* OR smartphone* OR cellphone* OR mobile* OR skype OR apps* OR ipad* OR multimedia* OR (multi W2 media*) OR video* OR ehealth)

\section{Mental health/Social isolation/Social interaction}

8. DE "OLDER people -- Social networks" DE "DISTRESS (Psychology) in old age" OR DE "GEROPSYCHOLOGY" OR DE "SOCIAL participation" OR DE "SOCIAL interaction" OR DE "SOCIAL isolation" OR DE "DISENGAGEMENT (Psychology)" OR DE "LONELINESS" OR DE "SOCIAL marginality" OR DE "ALIENATION (Social psychology)" OR DE "SELF-segregation (Sociology)" OR DE "SOCIAL distance" OR DE "SOCIAL invisibility" OR DE "SOCIAL anxiety" OR DE "MENTAL depression" OR DE "ANXIETY" OR DE "BOREDOM" OR DE "APATHY" OR DE "SADNESS" OR DE "HAPPINESS" OR DE "JOY" OR DE "MENTAL health" OR DE "DISCONTENT" OR DE "SATISFACTION" OR DE "WELL-being" OR DE "QUALITY of life" OR DE "SELF-efficacy in old age" OR DE "SELF-perception" OR DE "AGING -Psychological aspects" OR DE "LEISURE"

9. TI(particip* OR companion* OR leisure OR psychosocial OR (social W2 activit*) OR (social W2 behavior*) OR (social W2 activit*) OR (social W2 connect*) OR (social W2 engagement*) OR (social W2 involvement*) OR (social W2 inclusion*) OR (social W2 contact*) OR interact* OR "quality of life" OR (life N2 satisfaction*) OR wellbeing OR relax* OR happiness*OR (positive W2 emotion*) OR (social W2 identit*) OR "self efficacy" OR salutogen* OR (health W2 promotion) OR (health W2 prevention*) OR (illness W2 prevention) OR meaning OR distress* OR mood* OR loneliness OR boredom OR isolation* OR depress* OR anxiety) OR KW(particip* OR companion* OR leisure OR psychosocial OR (social W2 activit*) OR (social W2 behavior*) OR (social W2 activit*) OR (social W2 connect*) OR (social W2 engagement*) OR (social W2 involvement*) OR (social W2 inclusion*) OR (social W2 contact*) OR interact* OR "quality of life" OR (life N2 satisfaction*) OR wellbeing OR relax* OR happiness*OR (positive W2 emotion*) OR (social W2 identit*) OR "self efficacy" OR salutogen* OR (health W2 promotion) OR (health W2 prevention*) OR (illness W2 prevention) OR meaning OR distress* OR mood* OR loneliness OR boredom OR isolation* OR depress* OR anxiety) OR AB((psychosocial W2 intervent*) OR (social W2 activit*) OR (social W2 connect*) OR (social W2 behavior*) OR (social W2 connection*) OR (social W2 contact*) OR (social W2 interaction*) OR loneliness OR boredom OR isolation* OR "quality of life" OR (life N2 satisfaction*) OR wellbeing OR relax* OR happiness*OR (positive W2 emotion*) OR (social W2 identit*) OR "self efficacy" OR salutogen*OR (health W2 promotion*) OR (health W2 prevention*) OR (illness W2 prevention) OR distress*) 
Study types: Systematic reviews, meta-analysis, meta-synthesis

11. TX ((systematic* N3 review*) OR (metaanaly* OR (meta W2 analy*) OR (technology W2 overview*) OR

(technology W2 appraisal*) OR (technology W2 assessment*)) OR TX ((overview* N2 (review* OR systematic OR synthes* OR summary OR umbrella)) OR TX((integrative W3 review) OR (information W2 synthes*) OR (data W2 synthes*) OR (data N2 extract*)) OR JN ("Cochrane Database of Systematic Reviews") OR TX ((systematic W2 synthes*) OR (meta W2 synthes*) OR metasyntheses* OR (meta W2 ethnograph* OR metaethnograph* OR (thematic W2 synthes*) OR "meta-study" OR "meta-summary" OR "critical interpretive syntheses" OR "qualitative cross-case analysis" OR "grounded theory synthesis" OR "meta-interpretation"))

12. Limiters - Publication Type: Language: Danish, English, Norwegian, Swedish

13. TI ("young adults" OR "young people" OR child* OR adolescen* OR youth OR school* OR infant*)

\section{Combined sets}

\begin{tabular}{lc}
3 AND 4 & 147 \\
\hline $\mathbf{1 4}$ AND 11 & $\mathbf{1 8}$ \\
\hline 3 AND 7 AND 10 & $\mathbf{1 2 6 5}$ \\
\hline 16 AND 11 AND 12 & $\mathbf{1 0 0}$
\end{tabular}

The search result, usually found at the end of the documentation, forms the list of abstracts.

$\mathrm{AB}=$ Abstract

$\mathrm{AU}=$ Author

$\mathrm{DE}=$ Term from the thesaurus

$\mathrm{MM}=$ Major Concept

$\mathrm{TI}=$ Title

TX = All Text. Performs a keyword search of all the

ZC $=$ Methodology Index

$*$ = Truncation

$"$ " = Citation Marks; searches for an exact phrase

Social Services Abstracts/Sociological Abstracts via Proquest 2 September 2019

Search terms

Population: Age

1. (SU.EXACT("Elderly") OR SU.EXACT("Seniority") OR SU.EXACT("Retirement Communities") OR

SU.EXACT("Life Stage Transitions") OR SU.EXACT("Retirement") OR SU.EXACT("Pensions")) OR (ti(old*

OR elder* OR aged* OR aging* OR ageing* OR (old PRE/1 patient*) OR (old PRE/1 age*) OR (old* PRE/1 people*) OR (older PRE/1 patient*) OR adult* OR senior* OR retire*) OR if(old* OR elder* OR aged* OR aging* OR ageing* OR (old PRE/1 patient*) OR (old PRE/1 age*) OR (old* PRE/1 people*) OR (older PRE/1 patient*) OR adult* OR senior* OR retire*))

\section{Intervention: Social robots}

2. $\quad \mathrm{ti}\left(\left(\right.\right.$ companion N3 $\left._{\text {robot* }}^{*}\right)$ OR $\left(\right.$ social $^{*} \mathrm{~N} 3$ robot $\left.^{*}\right)$ OR (sociable N/2 robot*) OR (domestic PRE/2 robot*)

OR (healthcare N3 robot*) OR Paro OR (video PRE/2 game*) OR (assistive PRE/2 technolog*)) OR

$\mathrm{AB}$ ((companion N3 robot*) OR (social* N3 robot*) OR (sociable N/2 robot*) OR (domestic PRE/2 robot*) OR (healthcare N3 robot*) OR Paro OR (video PRE/2 game*) OR (virtual PRE/2 realit*) OR (virtual PRE/2 environment*) OR "science and technology studies" OR (assistive PRE/2 technolog*)) OR if((companion N3 robot*) OR (social* N3 robot*) OR (sociable N/2 robot*) OR (domestic PRE/2 robot*) OR (healthcare N3 robot*) OR Paro OR (video PRE/2 game*) OR (virtual PRE/2 realit*) OR (virtual PRE/2 environment*) OR "science and technology studies" OR (assistive PRE/2 technolog*))

\section{Intervention: ICT/Social media}

3. SU.EXACT("Information Technology") OR SU.EXACT("Telephone Communications") OR SU.EXACT("Internet") OR SU.EXACT("Virtual Reality") OR SU.EXACT("Electronic Technology")

4. $\quad$ ti((information N/2 technol*) OR ICT OR "science and technology studies" OR (virtual PRE/2 realit*) OR (virtual PRE/2 environment*) OR internet* OR computer* OR digital OR online OR (web PRE/2 based*) OR (web PRE/2 2.0) OR (social PRE/2 network*) OR twitter* OR facebook* OR "face book" OR blogging OR blogs OR (social PRE/2 media*) OR (electronic PRE/2 mail*) OR "e-mail" OR email* OR (text PRE/2 messag*) OR sms OR mms* OR phone* OR telephone* OR android* OR iphone* OR smartphone* OR cellphone* OR mobile* OR skype OR apps* OR ipad* OR multimedia* OR (multi PRE/2 media*) OR technolog* OR video* OR digital*) OR if((information N/2 technol*) OR "science and technology studies" OR (virtual PRE/2 realit*) OR ((virtual PRE/2 environment*) OR internet* OR computer* OR digital OR online OR (web PRE/2 based*) OR (web PRE/2 2.0) OR (social PRE/2 network*) OR twitter* OR facebook* OR "face book" OR blogging OR blogs OR (social PRE/2 media*) OR (electronic PRE/2 mail*) OR "e-mail" OR email* OR (text PRE/2 messag*) OR sms OR mms* OR phone* OR telephone* OR android* OR iphone* OR smartphone* OR cellphone* OR mobile* OR skype OR apps* OR ipad* OR multimedia* OR (multi PRE/2 media*) OR technolog* OR video*)) OR ab("science and technology studies" OR (virtual PRE/2 realit*) OR (virtual PRE/2 environment*) OR internet* OR computer* OR digital OR online OR web based* OR (social PRE/2 networking*) OR twitter* OR facebook* OR "face book" OR blogging OR blogs OR (social PRE/2 media*) OR (electronic PRE/2 mail*) OR "e-mail" OR email* OR (text PRE/2 messag*) OR sms OR mms* OR phone* OR telephone* OR android* OR iphone* OR smartphone* OR cellphone* OR (mobile PRE/2 phone*) OR skype OR apps* OR ipad* OR multimedia* OR (multi PRE/2 media*) OR (video PRE/2 game*))

$$
\text { 5. } 3 \text { OR } 4
$$


OR SU.EXACT("Loneliness") OR SU.EXACT("Social Interaction") OR SU.EXACT("Depression (Psychology)") OR SU.EXACT("Life Satisfaction") OR SU.EXACT("Well Being") OR SU.EXACT("Quality of Life")

7. ti(leisure OR companion* OR empower OR (psychosocial PRE/2 intervent*) OR (pro PRE/2 social) OR (social PRE/2 activit*) OR (social PRE/2 behavior*) OR (social PRE/2 connect*) OR (social PRE/2 contact*) OR (social PRE/2 interaction*) OR (social PRE/2 engagement*) OR (social PRE/2 involvement*) OR (social PRE/2 inclusion*) OR (social PRE/2 identit*) OR efficacy OR particip* OR loneliness OR boredom OR isolation* OR "quality of life" OR (life N/2 satisfaction*) OR wellbeing OR relax* OR happiness* OR (positive PRE/2 emotion*) OR meaning OR distress* OR mood* OR depress* OR anxiety OR salutogen* OR "health promotion" OR (health PRE/2 prevention*) OR (illness PRE/2 prevention)) OR if(leisure OR companion* OR empower OR (psychosocial PRE/2 intervent*) OR (pro PRE/2 social) OR (social PRE/2 activit*) OR (social PRE/2 behavior*) OR (social PRE/2 connectt*) OR (social PRE/2 contact*) OR (social PRE/2 interaction*) OR (social PRE/2 engagement*) OR (social PRE/2 involvement*) OR (social PRE/2 inclusion*) OR (social PRE/2 identit*) OR efficacy OR particip* OR loneliness OR boredom OR isolation* OR "quality of life" OR (life N/2 satisfaction*) OR wellbeing OR relax* OR happiness*OR (positive PRE/2 emotion*) OR meaning OR distress* OR mood* OR depress* OR anxiety OR salutogen* OR "health promotion" OR (health PRE/2 prevention*) OR (illness PRE/2 prevention))OR ab((psychosocial PRE/2 intervent*) OR companion* OR (social PRE/2 activit*) OR (social PRE/2 behavior*) OR (social PRE/2 connect*) OR (social PRE/2 contact*) OR (social PRE/2 interaction*) OR (social PRE/2 engagement*) OR (social PRE/2 involvement*) OR (social PRE/2 inclusion*) OR (social PRE/2 identit*) OR efficacy OR particip* OR loneliness OR boredom OR isolation* OR "quality of life" OR (life N/2 satisfaction*) OR wellbeing OR relax* OR happiness*OR (positive PRE/2 emotion*) OR distress* OR depress* OR anxiety OR salutogen* OR "health promotion" OR (health PRE/2 prevention*) OR (illness PRE/2 prevention))

8. 6 OR 7

Study types: Systematic reviews, meta-analysis, meta-synthesis

9. $\quad$ ti((systematic*N/3 review*) OR metaanaly* OR (meta PRE/2 analy*) OR (technology PRE/2 overview*) OR (technology PRE/2 appraisal*) OR (technology PRE/2 assessment*) OR (overview* N/2 (review* OR systematic OR synthes* OR summary OR umbrella)) OR (integrative PRE/2 review) OR (information PRE/2 synthes*) OR (data PRE/2 synthes*) OR (data N/2 extract*) OR (systematic PRE/2 synthes*) OR (meta PRE/2 synthes*) OR metasyntheses* OR (meta PRE/2 ethnograph*) OR metaethnograph* OR (thematic PRE/2 synthes*) OR "meta-study" OR "meta-summary" OR "critical interpretive syntheses" OR "qualitative crosscase analysis" OR "grounded theory synthesis" OR "meta-interpretation") OR if((systematic* N/3 review*) OR metaanaly* OR (meta PRE/2 analy*) OR (technology PRE/2 overview*) OR (technology PRE/2 appraisal*) OR (technology PRE/2 assessment*) OR (overview* N/2 (review* OR systematic OR synthes* OR summary OR umbrella)) OR (integrative PRE/2 review) OR (information PRE/2 synthes*) OR (data PRE/2 synthes*) OR (data N/2 extract*) OR (systematic PRE/2 synthes*) OR (meta PRE/2 synthes*) OR metasyntheses* OR (meta PRE/2 ethnograph*) OR metaethnograph* OR (thematic PRE/2 synthes*) OR "meta-study" OR "meta-summary" OR "critical interpretive syntheses" OR "qualitative cross-case analysis" OR "grounded theory synthesis" OR "meta-interpretation") OR ab ((systematic* N/3 review*) OR metaanaly* OR (meta PRE/2 analy*) OR (technology PRE/2 overview*) OR (technology PRE/2 appraisal*) OR (technology PRE/2 assessment*) OR (overview* N/2 (review* OR systematic OR synthes* OR summary OR umbrella)) OR (integrative PRE/2 review) OR (information PRE/2 synthes*) OR (data PRE/2 synthes*) OR (data N/2 extract*) OR (systematic PRE/2 synthes*) OR (meta PRE/2 synthes*) OR metasyntheses* OR (meta PRE/2 ethnograph*) OR metaethnograph* OR (thematic PRE/2 synthes*) OR "meta-study" OR "meta-summary" OR "critical interpretive syntheses" OR "qualitative cross-case analysis" OR "grounded theory synthesis" OR "meta-interpretation")

\section{Combined sets}

\begin{tabular}{lr}
1 AND 2 & 155 \\
\hline 10 AND 9 & $\mathbf{1 0}$ \\
\hline AND 5 AND 8 & 4681 \\
\hline 12 AND 9 & $\mathbf{1 6}$
\end{tabular}

The search result, usually found at the end of the documentation, forms the list of abstracts.

$\mathrm{AB}=$ Abstract

$\mathrm{AU}=$ Author

$\mathrm{DE}=$ Term from the thesaurus

$\mathrm{MM}=$ Major Concept

$\mathrm{TI}=$ Title

TX = All Text. Performs a keyword search of all the database's searchable fields

ZC $=$ Methodology Index

$*$ = Truncation

" " = Citation Marks; searches for an exact phrase

APPENDIX II - DESCRIBING THE INCLUDED REVIEWS, OBJECTIVES, INTERVENTION/OUTCOME, STUDY DESIGN AND NUMBER OF INCLUDED STUDIES, AS WELL AS MAIN RESULTS.

Reference: (Bleakley et al., 2015). Title: Gaming for health: a systematic review of the physical and cognitive effects of interactive computer games in older adults. Objective: Systematically review the evidence base and examine the physical and cognitive effects of physically based interactive com- puter games (ICG) in an older adult population. Also, how it affects user's compliance, enjoyment, and safety during exercise. Interventions: ICG and/or virtual reality (VR) technology supporting interaction with virtual objects in a computer-based environment. Interaction must involve strength, aerobic, balance, or flexibility components. Outcomes: cognitive or physical functioning. Secondary outcomes: adverse effects, enjoyment and compliance. Study design, number (n) 
of included studies: RCT $(n=5)$, observational $(n=5)$, controlled $(n=2)$. The review included 12 studies, published 2003-2010. Sample sizes (n): 6-40. Participants: mean age 65 years or older. Country (not applicable). Main results: Indications that ICG are safe and effective when used to promote physical activity among older adults. A small number of minor adverse events associated with ICG were reported. No evidence of an optimal exercise dose (intensity, exercise type or duration) was presented. There was a clear trend that balance could be positively strengthened by ICG. Findings mainly based on older participants (aged 63-96 years) with existing subsyndromal depression, showed some evidence that cognition, depressive symptoms, and quality of life could be positive influenced from ICG.

Reference: (Chen et al., 2016). Title: The effect of information communication technology interventions on reducing social isolation in the elderly: a systematic review. Objective: To explore the effects of ICT interventions on reducing social isolation of the elderly. Interventions: ICT interventions including for example webb - based apps, or via computers; as social networking sites, e-mail, online chat rooms, videoconferencing. Outcomes: Qualitative synthesis, with regard to social isolation, e.g. loneliness, belonging, fulfilling relationships, social contacts, quality of relationships, engagement, frequency, social network size and social activity. Study design, number (n) of included studies: RCT $(n=6)$, cohort study $(n=6)$, cross-sectional $(n=4)$, qualitative studies $(n=14)$. The review included 30 studies, based on 25 projects (5 studies reported 2 studies): published 2002-2015. Sample sizes (n) = 8-5203. Participants: mean range: $66-83$ years old (sometimes not determined). Countries: United Kingdom $(n=1)$, Canada $(n=1)$, Finland and Slovenia $(n=1)$, Sweden $(n=1)$, Norway $(n=1)$, Australia $(n=2)$, Israel $(n=2)$, New Zealand $(n=2)$, Taiwan $(n=2)$, Netherlands $(n=3)$, USA $(n=9)$. Main results: ICT use was consistently found to affect social support, social connectedness, and social isolation in general positively. The results for loneliness were inconclusive. The results for self-esteem and control over one's life were consistent but generally non-significant. Social isolation could be alleviated through four mechanisms: boosting self-confidence, connecting to the outside world, gaining social support, engaging in activities of interest. Some studies found a non-significant or negative impact, or that the positive effect of ICT use on social connectedness and social support did not last for more than six months after the intervention.

Reference: (Choi et. al., 2012). Title: Computer and internet interventions for loneliness and depression in older adults: a meta-analysis. Objec- tive: To examine the effectiveness of computer and Internet training interventions intended to reduce loneliness and depression in older adults. Interventions: Interventions involving computer or Internet use. Outcomes: Psychosocial outcomes, as loneliness and/or depression. Study design, number (n) of included studies: RCT $(n=4)$; quasi-experimental design $(n=2)$. The review included 6 studies published 2001-2012. Sample sizes (n): 26-109. Participants: 64-83 years old. Countries: Israel $(n=1)$, Netherlands $(n=2)$, USA $(n=3)$. Main results: The computer and Internet programs were effective in managing loneliness among older adults. Social support by use of computers and/or internet should be considered to help manage loneliness in this population. The overall mean weighted effect size for loneliness from five studies was statistically significant for decreased loneliness ( $Z$ $=2.085, p=0.037)$. The overall mean weighted effect size for depression from five studies was not statistically significant $(Z=1.528, p=0.126)$.

Reference: (Forsman, et. al., 2017). Title: Promoting mental wellbeing among older people: technology-based interventions. Objective: To explore the effectiveness of technology-based interventions in promoting the mental health and wellbeing of people aged 65 and over. Interventions: Technology-based interventions designed to promote mental wellbeing or independence. Outcomes: For example, aspects of quality of life associated with positive mental wellbeing, life satisfaction, empowerment, capability, civic participation, social resources and social inclusion. Study design, number (n) of included studies: $\operatorname{RCT}(n=6)$. The review included 21 articles, based on 19 evaluations of the effectiveness of technology-based interventions. Published 2003-2014. Sample sizes $(\mathbf{n})=5-335$. Participants: aged 65 years old or above, or 55 years old or more and retired. Countries: USA $(n=9)$, Spain $(n=2)$, Canada $(n=1), 1$ study respectively from Finland/Slovenia, Israel, Netherlands, Norway, Spain, Spain/Chile/Mexico/Cuba, Sweden and UK. English language publications (some in Spanish). Main results: Three out of six studies with high or moderate quality ratings (evaluating computer/internet training) reported statistically significant positive effects on psychosocial outcomes, including increased life satisfaction and experienced social support, as well as reduced depression levels among intervention recipients. Few randomized controlled trials, and the need for more methodologically rigorous studies are highlighted. Little comparability in outcome measures, resulting in an inconsistent evidence base with moderate strength and quality.

Reference: (Forsman \& Nordmyr 2017). Title: Psychosocial links between internet use and 
mental health in later life: a systematic review of quantitative and qualitative evidence. Objective: To summarize the evidence base of correlational data on Internet use and related psychosocial factors and its links to mental health among older adults, utilizing available data from both quantitative (NOTE. not presented in the present article) and qualitative studies. Interventions: Experiences of using computers and internet, internet use in general or for specific activities online, e-mail, dating sites, communication forums, online game, support via mail/ online communities, forums and instant messaging. Outcomes: Perceptions of psychosocial links between internet use and mental health. Quality of life, experienced loneliness, depressive symptoms and functional independence associated to mental health. Study design, number (n) of included studies: Focus groups $(n=2)$, qualitative interviews $(n=5)$, surveys $(n=4)$, ethnographical study (combination of observation and interview) $(n=2)$, qualitative (survey and interviews) $(n=1)$. The review included 14 studies published 2007-2013. Sample sizes (n): 7-1381. Participants: aged 60 years or more. Participants were required to be independent or assisted Internet users. Countries: Israel $(n=1)$, Spain $(n=1)$, Sweden $(n=1)$, UK $(n=2)$, Australia $(n=4)$, USA $(n=5)$. Main results: The review indicated support for links between Internet use and mental health and its psychosocial covariates in later life. Three themes emerged: New ways of communicating and connecting. The participants of the qualitative studies described how communication online (e- mail was the primary form of communication reported) supported maintained and increased contacts with family, friends, and others in their social network. The relationships were experienced as positive as well as influencing their subjective experience of mental health. An increase in resources according to needs and preferences. The Internet was experienced to provide and expand accessibility to resources other than those related to communication (e.g., public services, common interest groups). Internet use may support and enable participation in various activities and contexts chosen according to the older adults' preferences, thereby having an enabling and empowering effect. Mastering new technologies as a means of increased social inclusion. Limited technology experience or conventional attitudes may influence adoption of new technologies. Some participants expressed that the online world enhanced their mental health through promoting feelings of empowerment and improving self-esteem.

Reference: (Miller, et.al., 2014). Title: Effectiveness and feasibility of virtual reality and gaming system use at home by older adults for enabling physical activity to improve health related do- mains: a systematic review. Objective: To summarise evidence for the effectiveness and feasibility of VR/ gaming systems utilisation by older adults at home for enabling physical activity to improve impairments, activity limitations or participation. Interventions: Popular, commercially available gaming technologies were used to encourage physical activity in all but one of the reviewed studies. The Nintendo ${ }^{\mathrm{TM}}$ Wii, the $\mathrm{Wii}$ Sports package with the associated handheld remotes, fitness and strength games. Outcomes: Physical body functions or impairments, cognitive and emotional well-being. Participation and activity limitations. Study design, number (n) of included studies: case study $(n=7)$, controlled studies $(n=6)$ including two RCTs. no comparison $(n=1)$. The review included 14 studies published 2007-2012. Sample sizes (n): 1-60. Participants: majority of sample above 60 years old, mean age: 47-84 years. Countries (when applicable): North American $(n=7)$. Main results: The feasibility and effectiveness of VR/gaming systems used to enable physical activity in a home setting to address activity limitations, impairments and participation is relatively weak in this population. Insufficient evidence to provide sound recommendations for clinical practice, due to high risk of bias. The findings of this review may be used in planning future studies. When feasibility was addressed, strong retention and adherence with exercise was identified, as well as the need for assistance, training and monitoring to ensure safety for the users.

Reference: (Morris, et.al., 2014). Title: Smart technologies to enhance social connectedness in older people who live at home. Objective: To examine the effectiveness of smart technologies in improving or maintaining the social connectedness of older people living at home. Interventions: Smart technologies, including web-based information, intervention and communication programs. For example: online educational program, e-mail access to health professionals and online self-help programs, discussion forums, visual and verbal contact between study participants, training on computer, internet/e-mail use, use of pre-recorded, interactive telephone messages, the Nintendo Wii. The frequency of technology use varied. The programs lasted between four weeks and three years. Outcomes: Social connectedness (including social support, participation, empowerment, engagement, isolation and loneliness. Study design, number (n) of included studies: RCT $(n=12)$; cohorts $(n=6)$. The review included 18 studies published 2001-2011. Sample sizes (n): 12-39, Participants: mean age: 59-82 years. Countries: Norway $(n=1)$, Canada $(n=1)$, Netherlands $(n=4)$, USA $(n=11)$. Not available $(n=1)$. Main results: Fourteen studies reported significant positive outcomes in aspects 
of social connectedness such as social support, loneliness, social networks, empowerment and self-efficacy. All studies with positive results was based on online, interactive programs that incorporated support groups, health information, chat rooms or discussion boards. Implementation of technology to improve some dimensions of social connectedness is supported by emerging evidence. Also, smart technology might improve factors as quality of life, depression, self-esteem and stress. Many studies did not blind assessors or provide sufficient details about the intervention programs and/or participants. The risk of bias is thereby increased and might limit the generalization of findings to other contexts. In studies reporting outcomes related to social support $(n=6)$, the results were most consistent, and use of smart technology could help to achieve statistically significant improvements.

Reference: (Poscia, et al, 2018). Title: Interventions targeting loneliness and social isolation among the older people: an update systematic review. Objective: Aims to summarize and update the current knowledge on the effectiveness of the existing interventions for alleviating loneliness and social isolation among older persons. Interventions: The article included a variety of interventions, most of them not including digital tools, but four of them including interventions such as: CareTv for family contact among other features, computer use on a basic level, go online and use internet, social media, shopping, robot (Paro). Outcomes: Loneliness, well-being, feelings of safety, observation of social behaviour, quality of life for Alzheimers disease, social network. Study design, number (n) of included studies: Pre-post study $(n=3)$, RCT $(n=1)$. The review included 4 studies that evaluated the use of ICT, published 2012- 2015. Sample sizes (n): 35-85. Participants: aged between 55-100 years, mean age in one study 73, 1 years. Countries: New Zealand $(n=1)$, UK $(n=2)$, not specified $(n=1)$. Main results: Loneliness was significantly reduced in three out of four studies, and social network/social contacts were significantly improved. Well-being was improved but only in one primary study.

Reference: (Ronzi, et al, 2018). Title: What is the impact on health and wellbeing of interventions that foster respect and social inclusion in community - residing older adults? A systematic review of quantitative and qualitative studies. Objectives: The systematic review attempts to synthesise the evidence of health impacts of interventions on respect and social inclusion in older people. Interventions: Interventions for computer- technology use, learning supported by volunteers, computer - training program, including internet use, voice and video via internet information - and communication program. Outcomes: Depression, mental and physical health, anxiety, quality of life and wellbeing. Study design, number (n) of included studies: three quantitative (RCT $n=2$, controlled, $n=1$ ) and one qualitative (in-depth interviews) that evaluated the use of ICT, in total four studies. Published 2008-2012. Sample sizes (n): 7- 298. Participants: mean age between 64-81 years old, mean age 72 , or mean age 73 years. Countries: USA $(n=3)$, Netherlands $(n=1)$. Main results: Three studies concluded that depression was reduced, but not significantly following the intervention. Mental and physical health was not significantly improved. Quality of life and depressive symptoms were neither significantly improved. In the qualitative interview study participants reported experiences of enhanced wellbeing.

\section{APPENDIX III - EXCLUDED ARTICLES $(\mathrm{N}=76)$ BY REA- SON FOR EXCLUSION \\ Not a systematic review}

Beedholm, K., Frederiksen, K., \& Lomborg, K. (2016). What was also at stake when a robot bathtub was implemented in a Danish elder center: a constructivist secondary qualitative analysis. Qualitative health research, 26(10), 1424-1433.

Berner, J., Rennemark, M., Jogréus, C., \& Berglund, J. (2012). Distribution of personality, individual characteristics and internet usage in Swedish older adults. Aging \& mental health, 16(1), 119-126.

Cattan, M., Kime, N., \& Bagnall, A. M. (2011). The use of telephone befriending in low level support for socially isolated older people-an evaluation. Health \& social care in the community, 19(2), 198-206.

Gurley, K., Norcio, A. F. (2009) A systematic review of technologies designed to improve and assist cognitive decline for both the current and future aging populations. 3rd International Conference on Internationalization, Design and Global Development, IDGD 2009. Held as Part of $\mathrm{HCl}$ International 2009, San Diego, CA., p 156-163.

Ihm, J., \& Hsieh, Y. P. (2015). The implications of information and communication technology use for the social well-being of older adults. Information, communication \& society, 18(10), 1123-1138. Jongstra, S., Beishuizen, C., Andrieu, S., Barbera, M., van Dorp, M., van de Groep, B., \& Soininen, H. (2017). Development and validation of an interactive internet platform for older people: the healthy ageing through internet counselling in the elderly study. Telemedicine and e-Health, 23(2), 96-104.

Knapp, M., Perkins, M. (2010). The role befriending can play in older people's well-being. Community Care, 28-29.

Lampit, A., Valenzuela, M., \& Gates, N. J. (2015). Computerized cognitive training is beneficial for older adults. Journal of the American Geriatrics 
Society, 63(12), 2610-2612.

O'Brien N, Heaven B, Teal G, Evans EH, Cleland C, Moffatt S, \& Moynihan, P. (2016). Integrating evidence from systematic reviews, qualitative research, and expert knowledge using co-design techniques to develop a web-based intervention for people in the retirement transition. Journal of medical internet research, 18 (8), e210.

Preschl B, Wagner B, Forstmeier S, Maercker A (2011). E-health interventions for depression, anxiety disorder, dementia, and other disorders in older adults: A review. Journal of CyberTherapy and Rehabilitation, 4, 371-385.

Rodeschini, G. (2011). Gerotechnology: A new kind of care for aging? An analysis of the relationship between older people and technology. Nursing and health Sciences, 13 (4), 521-528.

Rogers, W. A., \& Fisk, A. D. (2010). Toward a psychological science of advanced technology design for older adults. Journals of gerontology Series B: Psychological sciences and social sciences, 65(6), 645-653.

Roh, S., Lee, Y. S., Kim, Y., Park, S. Y., \& Chaudhuri, A. (2015). Gender differences in the roles of religious support and social network support in reducing depressive symptoms among older Korean Americans. Journal of social service research, 41(4), 484-497.

Sánchez Criado, T., Domènech, M. (2015). Older people in a connected autonomy? Promises and challenges in the technologisation of care. ¿Personas mayores en autonomía conectada? Promesas y retos en la tecnologización del cuidado. Revista Española de Investigaciones Sociológicas, 152, 105-120.

Scanlon, L., O'Shea E., O'Caoimh, R., Timmons, S. (2015). Technology use and frequency and self-rated skills: A survey of community-dwelling older adults. Journal of the American geriatrics society, 63 (7), 1483-1484.

Warburton, J., Cowan, S., Winterton, R., Hodgkins, S. (2014). Building social inclusion for rural older people using information and communication technologies: Perspectives of rural practitioners.

Australian social work, 67(4), 479-494.

Vichitvanichphong, S., Talaei-Khoei, A., Kerr, D., Ghapanchi, A. H., \& Scott-Parker, B. (2016). Good Old Gamers, Good Drivers: Results from a correlational experiment among older drivers. Australasian journal of information systems, 20.

\section{Wrong intervention}

Adamek, M. E, Slater, G.Y. (2008). Depression and anxiety. Journal of gerontological social work, 50, 153-189.

Bolle, S., van Weert, J. C., Daams, J. G., Loos, E. F., De Haes, H. C., \& Smets, E. M. (2015). Online health information tool effectiveness for older patients: A systematic review of the literature. Journal of health communication, 20(9), 1067-1083.
Coll-Planas, L., Nyqvist, F., Puig, T., Urrútia, G., Solà, I., \& Monteserín, R. (2017). Social capital interventions targeting older people and their impact on health: a systematic review. Journal of epidemiol Community Health, 71(7), 663-672. Demiris G, Hensel BK. (2008) Technologies for an aging society: a systematic review of "smart home" applications. Yearb Med Inform, 33-40. Klainin-Yobas, P., Oo, W. N., Suzanne Yew, P. Y., \& Lau, Y. (2015). Effects of relaxation interventions on depression and anxiety among older adults: a systematic review. Aging \& mental health, 19(12), 1043-1055.

Liu, L., Stroulia, E., Nikolaidis, I., Miguel-Cruz, A., \& Rincon, A. R. (2016). Smart homes and home health monitoring technologies for older adults: A systematic review. International journal of medical informatics, 91, 44-59.

Ludwig, W., Wolf, K. H., Duwenkamp, C., Gusew, N., Hellrung, N., Marschollek, M., \& Haux, R. (2012). Health-enabling technologies for the elderly-an overview of services based on a literature review. Computer methods and programs in biomedicine, 106(2), 70-78.

Medical Advisory Secretariat. (2008) Social isolation in community-dwelling seniors: an evidence-based analysis. Ontario health technology assessment series, 8, 1-49.

Peek, S.T., Wouters, E.J., van Hoof, J., Luijkx, K. G., Boeije, H. R., Vrijhoef, H. J. (2014). Factors influencing acceptance of technology for aging in place: a systematic review. International journal of medical informatics, 83(4), 235-248.

Reeder, B., Meyer, E., Lazar, A., Chaudhuri, S., Thompson, H. J., Demiris, G. (2013). Framing the evidence for health smart homes and homebased consumer health technologies as a public health intervention for independent aging: a systematic review. International journal of medical informatics, 82 (7), 565-579.

Shvedko, A., Whittaker, A.C., Thompson, J. L., Greig, C. A. (2018). Physical activity interventions for treatment of social isolation, loneliness or low social support in older adults: A systematic review and meta-analysis of randomised controlled trials. Psychology of sport and exercise, 34, 128-137.

Song, Y., van der Cammen, T. J. M. (2019). Electronic assistive technology for community-dwelling solo-living older adults: A systematic review. Maturitas, 125, 50-56.

Rosenbaum, S., \& Sherrington, C. (2011). Is exercise effective in promoting mental well-being in older age? A systematic review. British journal of sports medicine, 45(13), 1079-1080.

Winterton, R., Warburton, J. (2011). Models of care for socially isolated older rural carers: barriers and implications. Rural \& remote health, 11, 1-13

\section{Wrong outcome}

Baig, M. M., Afifi, S., GholamHosseini, H., \& Mirza, F. (2019). A systematic review of wear- 
able sensors and loT-based monitoring applications for older adults-a focus on ageing population and independent living. Journal of medical systems, 43(8), 233.

Baker, S., Warburton, J., Waycott, J., Hoang, T., Dow, B., Ozanne, E., Vetere F. (2018) Combatting social isolation and increasing social participation of older adults through the use of technology: A systematic review of existing evidence. Australasian journal on Ageing, 37 (3), 184-193. Cacciata, M., Stromberg, A., Lee, J-A., Sorkin, D., Lombardo, D., Clancy, S., Nyamathi, A., Evangelista, L. S. (2019). Effect of exergaming on healthrelated quality of life in older adults: A systematic review. International journal of nursing studies, 93, 30-40.

Campos, W., Martinez, A., Sanchez, W., Estrada, H., Castro-Sánchez, N. A., \& Mujica, D. (2016). A systematic review of proposals for the social integration of elderly people using ambient intelligence and social networking sites. Cognitive computation, 8(3), 529-542.

Dickens, A.P., Richards, S.H., Greaves, C.J., Campbell, J. L. (2011) Interventions targeting social isolation in older people: a systematic review. BMC Public Health, 11(1), 647.

Fischer, S.H., David, D., Crotty, B. H., Dierks M., Safran, C. (2014). Acceptance and use of health information technology by communitydwelling elders. International journal of medical informatics, 83(9), 624-635.

Hodgetts, S., Gallagher, P., Stow, D., Ferrier, I. N, O'Brien JT. (2017). The impact and measurement of social dysfunction in late-life depression: an evaluation of current methods with a focus on wearable technology. International journal of geriatrics psychiatry, 32(3), 247-55.

Neri, S. G. R., Cardoso, J.R., Cruz, L., Lima, R.M., De Oliveira, R.J., Iversen, M. D., Carregaro, R., L. (2017). Do virtual reality games improve mobility skills and balance measurements in community-dwelling older adults? Systematic review and meta-analysis. Clinical Rehabilitation, 31(10), $1292-1304$.

Pearce, A. J., Adair, B., Miller, K., Ozanne, E., Said, C., Santamaria, N., \& Morris, M. E. (2012). Robotics to enable older adults to remain living at home. Journal of aging research, 2012, 538-169. Pedrozo Campos Antunes, T., Souza Bulle de Oliveira, A., Hudec, R., Brusque Crocetta, T., Ferreira de Lima Antão, J. Y., de Almeida Barbosa, R. T., \& de Abreu, L. C. (2019). Assistive technology for communication of older adults: a systematic review, Aging \& mental health, 23 (4), 417-427.

Penteridis, L., D'Onofrio, G., Sancarlo, D., Giuliani, F., Ricciardi, F., Cavallo, F. \& Gkiokas, A. (2017). Robotic and sensor technologies for mobility in older people. Rejuvenation research, 20(5), 401-410.

Shishehgar, M., Kerr, D., Blake J. (2019). A systematic review of research into how robotic technology can help older people. Smart health, 7-8, 1-18.

Skjaeret, N., Nawaz, A., Morat, T., Schoene, D., Helbostad, J. L., Vereijken, B. (2016). Exercise and rehabilitation delivered through exergames in older adults: An integrative review of technologies, safety and efficacy. International journal of medical informatics, 85(1), 1-16.

Stanmore, E., Stubbs, B., Vancampfort, D., de Bruin ED., Firth J. (2017).

The effect of active video games on cognitive functioning in clinical and non-clinical populations: A meta-analysis of randomized controlled trials. Neuroscience \& Biobehavioral Reviews, 78, 34-43

Taylor, L.M, Kerse, N., Frakking, T., Maddison, R. (2018). Active video games for improving physical performance measures in older people. A meta-analysis. Journal of Geriatric Physical Therapy, 41 (2), 108-123

Vázquez, F. L., Otero, P., García-Casal, J. A., Blanco, V., Torres, Á. J., \& Arrojo, M. (2018). Efficacy of video game-based interventions for active aging. A systematic literature review and meta-analysis. PloS one, 13(12), e0208192.

Van de Meulebroucke T, de Casterlé, B. D, Gastmans, C. (2018). How do older adults experience and perceive socially assistive robots in aged care: a systematic review of qualitative evidence. Aging \& mental health, 22 (2), 149-167.

\section{Wrong intervention/outcome}

Vichitvanichphong, S., Talaei-Khoei, A., Kerr, D., \& Ghapanchi, A. H. (2018). Assistive technologies for aged care: Comparative literature survey on the effectiveness of theories for supportive and empowering technologies. Information technology \& People, 31 (2), 405-427.

\section{Wrong population}

Abbott, R., Orr, N., McGill, P., Whear, R., Bethel, A., Garside, R., \& Thompson-Coon, J. (2019). How do "robopets" impact the health and wellbeing of residents in care homes? A systematic review of qualitative and quantitative evidence. International journal of older people nursing, 14(3), e12239.

Bech Mikkelsen, A. S., Petersen, S., Dragsted, A. C., Kristiansen, M. (2019) Social interventions targeting social relations among older people at nursing homes: A qualitative synthesized systematic review. INQUIRY: The Journal of Health Care Organization, Provision, and Financing, 56, 1-16. Bemelmans, R., Gelderblom, G. J., Jonker, P., \& De Witte, L. (2012). Socially assistive robots in elderly care: a systematic review into effects and effectiveness. Journal of the American medical directors association, 13(2), 114-120.

Bhattarai, P., \& Phillips, J. L. (2017). The role of digital health technologies in management of pain in older people: An integrative review. Ar- 
chives of gerontology and geriatrics, 68, 14-24. Boots LMM, de Vugt ME, van Knippenberg RJM, Kempen GIJM, Verhey FRJ. (2014) A systematic review of internet-based supportive interventions for caregivers of patients with dementia. Int J Geriatr Psychiatry, 29, 331-44.

Brijnath, B., Protheroe, J., Mahtani, K. R., Antoniades, J. (2016) Do Web-based mental health literacy interventions improve the mental health literacy of adult consumers? Results from a systematic review. Journal of medical internet research, 18, e165.

Chen, S-C., Jones, C., Moyle, W. (2018). Social robots for depression in older adults: A systematic review. Journal of Nursing Scholarship, 50, 612-622.

Dove, E., Astell, A. J. (2017). The use of motionbased technology for people living with dementia or mild cognitive impairment: A literature review. Journal of medical internet research, 19, e3. Góngora Alonso, S., Hamrioui, S., de la Torre Díez, I., Motta Cruz, E., López-Coronado, M., Franco, M. (2019). Social robots for people with aging and dementia: A systematic review of literature. Telemedicine and health, 25, 533-540.

Hill, N. T., Mowszowski, L., Naismith, S. L., Chadwick, V. L., Valenzuela, M., \& Lampit, A. (2017). Computerized cognitive training in older adults with mild cognitive impairment or dementia: a systematic review and meta-analysis. American journal of psychiatry, 174 (4), 329-340. Holthe, T., Halvorsrud, L., Karterud, D., Hoel, K. A., \& Lund, A. (2018). Usability and acceptability of technology for community-dwelling older adults with mild cognitive impairment and dementia: a systematic literature review. Clinical interventions in aging, 13, 863- 886.

Huang, C. (2010). Internet use and psychological well-being: A meta-analysis. Cyberpsychology, Behavior, and Social Networking, 13(3), 241-249. Kachouie, R., Sedighadeli, S., Khosla, R., \& Chu, M. T. (2014). Socially assistive robots in elderly care: a mixed-method systematic literature review. International journal of human-computer interaction, 30(5), 369-393.

Kampmeijer, R., Pavlova, M., Tambor, M., Golinowska. S., Groot, W. (2016) The use of ehealth and $\mathrm{m}$-health tools in health promotion and primary prevention among older adults: a systematic literature review. BMC health services research, 16, 290.

Kapadia, V., Ariani. A., Li, J., Ray, P. K. (2015). Emerging ICT implementation issues in aged care. International journal of medical informatics, 84, 892-900.

Lazar, A., Thompson, H., Demiris, G. (2014). A systematic review of the use of technology for reminiscence therapy. Health education and behavior, 41, 51s-61s.

Liu, D., \& Baumeister, R. F. (2016). Social networking online and personality of self-worth: A meta-analysis. Journal of research in personality, 64, 79-89.

Mancioppi, G., Fiorini, L., Timpano Sportiello, M., \& Cavallo, F. (2019). Novel technological solutions for assessment, treatment, and assistance in mild cognitive impairment: a systematic review. Frontiers in neuroinformatics, 13, 58.

Mitchell, J. I., Long, J. C., Braithwaite, J., \& Brodaty, H. (2016). Social-professional networks in long-term care settings with people with dementia: an approach to better care? A systematic review. Journal of the American medical directors association, 17(2), 183.e17-183.e27.

Neville, L. M., O'Hara, B., Milat., A. (2009). Computer-tailored physical activity behavior change interventions targeting adults: A systematic review. International journal of behavioral nutrition and physical activity, 6 1), 30.

Newlin, M., Webber, M., Morris, D., Howarth, S. (2015). Social participation interventions for adults with mental health problems: A review and narrative synthesis. Social work research, 39 (3), 167-180.

Pallavicini, F., Ferrari, A., Mantovani, F. (2018). Video games for well-being: A systematic review on the application of computer games for cognitive and emotional training in the adult population. Frontiers in psychology, 9, 2127.

Primack, B. A., Carroll, M. V., McNamara, M., Klem, M. L., King, B., Rich, M, \& Nayak, S. (2012). Role of video games in improving health-related outcomes: A systematic review. American journal of preventive medicine, 42 (6), 630-638.

$\mathrm{Pu}$, L., Moyle, W., Jones, C., Todorovic, M. (2019). The effectiveness of social robots for older adults: A systematic review and meta-analysis of randomized controlled studies. The Gerontologist, 59(1), e37-e51.

Robinson, N. L, Cottier, T. V., Kavanagh, D. J. (2019). Psychosocial health interventions by social robots: Systematic review of randomized controlled trials. Journal of medical internet research, 21(5), e13203.

Street, T. D., Lacey, S. J., Langdon, R. R. (2017). Gaming your way to health: A systematic review of exergaming programs to increase health and exercise behaviors in adults. Games for Health Journal, 6(3), 136-146.

Teychenne, M., Ball, K., Salmon. J. (2010). Sedentary behavior and depression among adults: A review. International journal of behavioral medicince, 17(4), 246-254.

\section{APPENDIX IV - SySteMATIC REVIEWS WITH HIGH RISK OF BIAS, AND LOW QUALITY ACCORDING TO AM- STAR ( $=16)$. *No ANSWER TO AMSTAR QUES- TION NUMBER LISTED.}

Blaschke, C. M., Freddolino, P. P., Mullen, E. E. (2009). Ageing and technology: A review of the research literature. British journal of social work, 39 (4), 641-656. *2, 3, 6, 7, 10, 11 
Brimelow, R.E., Wollin, J.A. (2017). Loneliness in old age: Interventions to curb loneliness in longterm care facilities. Activities, adaptation \& aging, 41(4), 301-315. *2, 7, 9, 10

Broekens, J., Heerink, M., Rosendal, H. (2009). Assistive social robots in elderly care: A review. Gerontechnology, 8 (2), 94-103. *6, 7, 8, 10, 11 Chao YY, Scherer YK, Montgomery CA. (2015). Effects of using Nintendo Wii exergames in older adults: a review of the literature. Journal of aging and health, 27(3), 379-402.* 2, 3, 7, 8

Cohen-Mansfield, J., Perach, R. (2015). Interventions for alleviating loneliness among older persons: a critical review. American Journal of health promotion, 29(3), e109-e125. *2, 3, 7, 8, 10, 11

Damant, J., Knapp, M., Freddolino P., Lombard, D. (2016). Effects of digital engagement on the quality of life of older people. Health and social care in the community, 25(6), 1679-1703. *2, 3, $7,8,10,11$

Hagan, R., Manktelow, R., Taylor, B. J., Mallett, J. (2014). Reducing loneliness amongst older people: a systematic search and narrative review. Aging and mental health, 18(6), 683-693. *2, 7, 8, 10, 11 Hall, A. K., Chavarria, E., Maneeratana, V., Chaney, B. H., Bernhardt, J. M. (2012). Health benefits of digital videogames for older adults: A systematic review of the literature. Games for health journal, 1(6), 402-410. *7, 8, 10

Khosravi, P., Ghapanchi, A. H. (2015). Investigating the effectiveness of technologies applied to assist seniors: A systematic literature review. International journal of medical informatics, 85(1),
17-26. *3, 6, 7, 8, 10, 11

Khosravi, P., Rezvani, A., Wiewiora, A. (2016). The impact of technology on older adults' social isolation. Computers in Human Behavior, 63, 594-603. *3, 6, 7, 8, 10, 11

Li, J., Theng, Y.L., Foo, S. (2016). Effect of exergames on depression: A systematic review and meta-analysis. Cyberpsychology, behavior, and social networking, 19(1), 34-42. *2, 7, 8, 10, 11

Li. J., Erdt, M., Chen, L., Cao, Y., Lee, S-Q., Theng, Y-L. (2018). The social effects of exergames on older adults: Systematic review and metric analysis. Journal of medical internet research, 20(6), e10486. *2, 7, 9,10

Marston, H. R, Smith, S. T. (2012). Interactive videogame technologies to support independence in the elderly: A narrative review. Games for health: Research, Development, and Clinical Applications, 1 (2), 139-152. *2, 7, 8, 10, 11

Nef, T., Ganea, R. L., Müri, R. M., Mosimann, U. P. (2013). Social networking sites and older users: A systematic review. International psychogeriatrics, 25(7), 1041-1053. *7, 8, 10

Shishehgar, M., Kerr, D., Blake. J. (2019). The effectiveness of various robotic technologies in assisting older adults. Health informatics journal, 25(3), 892-918. *2, 6, 7, 9, 10

Verheijden Klompstra L, Jaarsma T, Strömberg A. (2014). Exergaming in older adults: A scoping review and implementation potential for patients with heart failure. European journal of cardiovascular nursing, 13(5), 388-398. *4, 7, 8,10 